\title{
Thermophilous fringe communities as an indicator of vegetation changes: a case study of the "Murawy Dobromierskie" steppe reserve (Poland)
}

\author{
Tomasz Szygendowski ${ }^{*}$ \& Andrzej Brzeg ${ }^{2}$
}

\author{
${ }^{1}$ Osiedle Pod Lipami 3/55, 61-629 Poznań, Poland \\ ${ }^{2}$ Department of Plant Ecology and Environmental Protection, Faculty of Biology, Adam Mickiewicz University, Umultowska 89, 61-614 \\ Poznań, Poland \\ * corresponding author (e-mail: szygend@gmail.com)
}

\begin{abstract}
In this paper, changes of the non-forest xerothermic vegetation of the "Murawy Dobromierskie" steppe reserve which occurred in the period 1993-2012 are examined. The material comprises 50 relevés, of which 43 date from 2012 and the other 7 - from 1993. Reléves were arranged in 5 analytic tables. A synoptic table was also compiled, and for each syntaxonomical species group distinguished, values of the cover coefficient (C), the collective group share index (G), and the systematic group value (D) were estimated and compared. On the basis of the obtained results, a significant decline in abundancy and/or constancy was observed within the following groups: Ch. Artemisietea vulgaris, Ch. Cirsio-Brachypodion pinnati, Ch. Festuco-Brometea, Ch. Geranion sanguinei, Ch. Koelerio-Corynephoretea, and Ch. Origanetalia and Trifolio-Geranietea sanguinei, whereas for the taxa of the Rhamno-Prunetea, a notable increase in the share of the reserve vegetation was recorded. A sizeable expansion of the moss layer was also observed in this period. The results are discussed with special regard to differences in the methodical background of both field studies.
\end{abstract}

Key words: secondary succession, xerothermic grasslands, numerical parameters, steppe reserve, Festuco-Brometea, TrifolioGeranietea sanguinei, Małopolska Upland, Poland

\section{Introduction}

Xerothermic grasslands of the Festuco-Brometea class in Central and Western Europe are mostly extrazonal, "steppe-type" vegetation patches, either of semi-natural (anthropogenic) or relic character (Medwecka-Kornaś \& Kornaś 1972; Ellenberg 1996). A massive decline of this habitat type, ongoing since the end of $19^{\text {th }}$ century, has its origin, on the one hand, in land-use intensification and, on the other, in pasture abandonment, which has led to their rapid secondary succession (Willems 2001; Poschlod \& WallisDe Vries 2002). As early as in 1920s, it was reported that the latter process was occurring on a large scale (Kozłowska 1925). In the course of later investigations, various issues concerning overgrowing of dry grasslands were studied, e.g.: succession-driven changes of species richness, floristic composition, and community structure; successional stages and species turnover; limitation of microsites and seedling dispersal; and methods of effective restoration of patches (Faliński 1972; Bobbink \& Willems 1987, 1993; Świerczyńska 1990; Wilmanns \& Sendtko 1995; Zerbe \& Schacht 1998; Kupferschmid et al. 2000; Bąba 2003; Dierschke 2006; Chytrý 2007; Enyedi et al. 2008; Schrautzer et al. 2009; Rusina \& Kiehl 2010; Hahn et al. 2012; Hegedüšová Vantarová \& Škodová 2014). Some studies showed that, except for trees and shrubs, calcareous grasslands can also be encroached with tall herbs, forming patches of xerothermophilous communities of the Trifolio-Geranietea sanguinei (Ger."Versaumung") class. Such communities should be regarded as post-terminal successional stages of abandoned calcareous grasslands (Dierschke 1985, 2006; Wilmanns \& Kratochwil 1983; Kratochwil 1984; Brzeg 2005; Chytrý 2007; Brzeg \& Wika 2014).

In Poland, however, such herb phytocoenoses were, for a long time, classified as specific or "successional" forms of Festuco-Brometea communities (e.g. 
Medwecka-Kornaś \& Kornaś 1963, 1972; Grodzińska 1970 ), contributing to a poor state of their recognition. According to the first vegetation diagnosis of the "Murawy Dobromierskie" steppe reserve (Małopolska Upland) by Olaczek et al. (1993), the most widespread non-forest plant community type was the xerothermic grassland association Thalictro-Salvietum pratensis Medw.-Korn. 1959. At the same time, the above-mentioned authors emphasized that, in terms of its origin, the object vegetation was fully anthropogenic and, since the plantation of pine in 1950s, followed by abandonment of pastures, it was undergoing rapid secondary succession changes. As a matter of fact, those ThalictroSalvietum pratensis phytocoenoses were often found in their "post-terminal" development phase (Olaczek et al. 1993). It is highly probable though, that most of such patches actually represented fringe communities of the Trifolio-Geranietea sanguinei. The last research on the reserve calcareous open-area vegetation showed that it was dominated by phytocoenoses of four associations of the Geranion sanguinei alliance, Trifolio-Geranietea sanguinei class (Szygendowski 2013).

A major focus of this paper is, thus, laid on comparing the state of the object non-forest xerothermic vegetation in the years 1993 and 2012 in order to analyze the secondary succession process in the syndynamical aspect. Moreover, the work aims to characterize rare and valuable forb fringe communities of the Geranion sanguinei documented in the course of the last survey (Szygendowski 2013).

\section{Research area}

The reserve (Fig. 1) covers an area of 36.29 ha. It is located about $2 \mathrm{~km}$ east of the village of Dobromierz $\left(51^{\circ} 00^{\prime} 32^{\prime \prime} \mathrm{N} 19^{\circ} 55^{\prime} 18^{\prime \prime} \mathrm{E}\right)$, in Kluczewsko municipality, Włoszczowa district, Świętokrzyskie Voivodship, within the area of Przedbórz Landscape Park. The reserve is situated in the Małopolska Upland sub-province, the Przedbórz Upland (342.1) macroregion, in the northwestern part of the Przedbórz-Małogoszcz Range (342.15) mesoregion (Kondracki 1998). Its area occupies southfacing slopes and summits of a Jurassic limestone hill. The most frequently occurring soil type is rendzina, turning into brown soils and - much more rarely - brown arenosol on sandy deposits at the foot of the hill. Average $\mathrm{pH}$ level ranges from 5.5-7.5 for rendzina to 4.5-5.0 for brown arenosols (Olaczek et al. 1993).

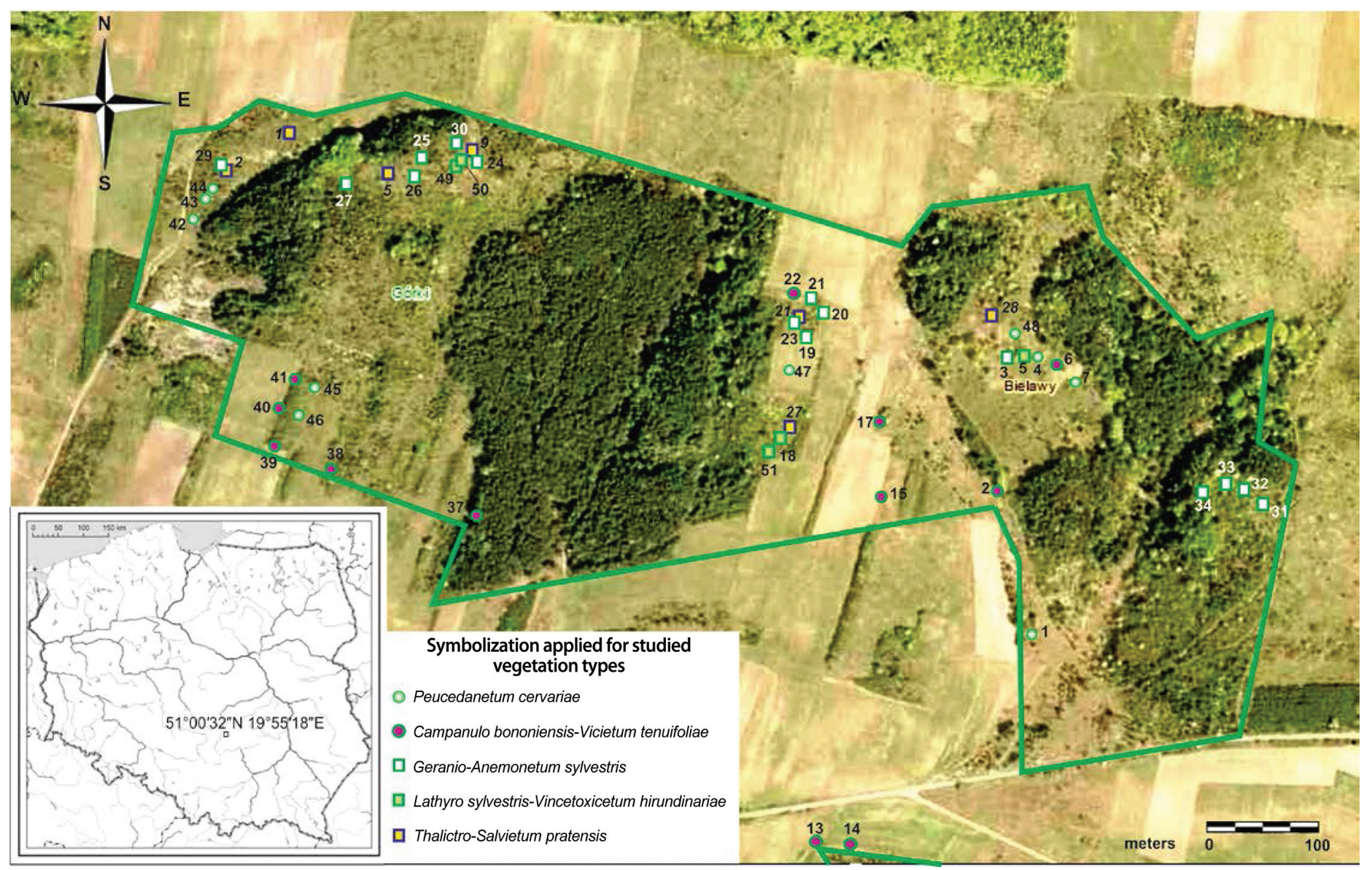

Fig. 1. Geographical location of the study area and distribution of relevés in the "Murawy Dobromierskie" reserve Source: Esri (2014) World Topographic Map. Category: imagery with labels. 1 : 6,850. Copyright by Esri, DeLorme, HERE, TomTom [accessed 2014-03-01] 
The area of today's reserve Murawy Dobromierskie has been recognized as a refuge of xerothermic flora since early 1980s (Wnuk 1981, 1984). The first full list of the reserve flora was drawn up by Olaczek et al. (1993). About 320 species of vascular plants and 10 species of mosses were found in the reserve area at that time. At the present time, 14 plant species under protection (pursuant to the Regulation 2014) are known to occur in the object.

Based on the first reserve vegetation diagnosis by Olaczek et al. (1993), the existence of 12 associations pertaining to 6 classes was stated. The authors documented the following community types occurring in the area: psammophilous swards ( 1 rel.), calcareous grasslands (14 rel.), xerothermic shrubs (7 rel.), forests (14 rel.), and segetal communities (21 rel.). Some grassland patches with the greatest abundance of Aster amellus were further recognized as a marginal form of the Inuletum ensifoliae association (Wnuk \& Pisarek 2008)

\section{Material and methods}

Field studies were carried out in 2012. At the stage of data collection, the Braun-Blanquet approach (BraunBlanquet 1964; Pawłowski 1972; Matuszkiewicz 2001; Dzwonko 2007) was applied. Areas of relevés ranged from 8 to $50 \mathrm{~m}^{2}$; their typical localization was shrub/ forest edges, formerly cultivated open areas and/or former limestone excavations. To obtain a good insight into syntaxonomic differentiation of the reserve vegetation, and to avoid involvement of vegetation mosaic (i.e. grassland accompanied by herbs and shrubs, see Müller 1962, 1978) in single relevés, the authors regarded selection of homogenous patches as one of the major principles of field work, so that relevés did not exceed $50 \mathrm{~m}^{2}$ in area. In another study, however, (Olaczek et al. 1993), the area of relevés ranged from 100 to 400 $\mathrm{m}^{2}$ indicating a higher homogeneity of vegetation. To provide comparability of two data sets, the authors ensured that the total area of the compared relevé sets was nearly the same (7 relevés in $1993-750 \mathrm{~m}^{2}$ and 43 relevés in $2012-761 \mathrm{~m}^{2}$ ).

For each of the distinguished 4 associations, an analytic phytosociological table was assembled. A separate analytic table of the previously documented calcareous grassland association (Thalictro-Salvietum pratensis) was compiled, and it comprised 7 relevés randomly selected from the original data collected by Olaczek et al. (1993). In a classical synoptic table, all the associations analyzed in the paper are compared. The category tagged G.s. coll. took all the examined associations of thermophilous herbs (i.e. of the Geranion sanguinei alliance) as a whole, and enabled to better illustrate the state of the entire xerothermic non-forest vegetation of the reserve in year 2012. Spatial distribution of all relevés compared in the present paper is illustrated in Figure 1. The background was derived from the Esri ${ }^{\circledR}$ World Topographic Map.

In order to elucidate the main successional trends ongoing in the Murawy Dobromierskie reserve, a comparative table of three parameters: total mean cover coefficient $(\mathrm{C})$, collective group share index $(\mathrm{G})$, and systematic group value (D), estimated for the distinguished groups of taxa, was compiled. The cover coefficients and constancy degrees were calculated with the use of ${ }^{\circ}$ ExGrad for Windows 1.01 (Sławnikowski 1998) software. In each analytic table, the obtained results were added up within all syntaxonomical groups. For other estimations, MS Excel software was used. The D values were calculated by the formula: $D=G^{*} S / 100$, where $\mathrm{G}$ (collective group share index) and $\mathrm{S}$ (group constancy) were calculated as follows: $G=(g / t) 100 ; S$ $=\left(\mathrm{g} / \mathrm{z}^{*} \mathrm{n}\right) 100$. The following designations were useds: $\mathrm{g}$ - the sum of occurrences of a given syntaxonomical group in the analytic table; $t$ - the sum of occurrences of all taxa in the table; $\mathrm{z}$ - the number of taxa of a given group; $\mathrm{n}$ - the number of relevés in the table. For four Geranion sanguinei associations taken collectively, the $\mathrm{C}$ coefficient sums, the $\mathrm{G}$ indices, and the $\mathrm{D}$ values, obtained for each group of taxa, were averaged. In the calculation of the parameters, only vascular plant taxa were taken into account.

To precisely indicate the syntaxonomical status of the taxa listed in each analytic table, the following abbreviations were applied: A - Artemisietea vulgaris; B - Brometalia erecti; C-B - Cirsio-Brachypodion pinnati; F-B -Festuco-Brometea; $\mathrm{G}$ - Geranion sanguinei; K-C - Koelerio-Corynephoretea; M-A - MolinioArrhenatheretea; O - others; Q-F - Querco-Fagetea; $\mathrm{R}-\mathrm{P}$-Rhamno-Prunetea; V-P - Vaccinio-Piceetea. The nomenclature of syntaxonomical units is compatible with current classification by Ratyńska et al. (2010).

The nomenclature of vascular plants and mosses follows works by Rutkowski (2004) and Ochyra et al. (2003), respectively. In the 2013 study, the taxonomic approach to species was more detailed than the one applied in the preceding research, and the following microtaxa were distinguished: Achillea millefolium s.s. and A. collina (vs. A. millefolium s.l), none of which was taken into account in the comparison; besides: Leontodon hispidus s.s. and L. hispidus ssp. danubialis (vs. $L$. hispidus s.1.); Pimpinella saxifraga s.s. and P. nigra (vs. P. saxifraga s.1.); Poa angustifolia var. setacea (vs. P. angustifolia s.1.); Pyrus pyraster (vs. P. communis s.1.); and Thymus pannonicus s.s. and Th. pannonicus ssp. marschallianus (vs. Th. pannonicus s.1.), which all were treated as aggregate species. Furthermore, two species: Polygala comosa and P. vulgaris (the occurence of the latter on dry chalk sites seems very controversial) were regarded in the comparison as a single unit. 


\section{Results}

\subsection{Differentiation of the contemporary vegetation}

\subsubsection{Syntaxonomical classification of the distinguished units}

At the present time, open areas of the steppe reserve "Murawy Dobromierskie" are overgrown predominantly by the vegetation representing the Geranion sanguinei R.Tx. in Th. Müller 1962 alliance, belonging to the Origanetalia vulgaris Th. Müller 1962 order and to the Trifolio-Geranietea sanguinei Th. Müller 1962 class. The research from 2012 showed the existence of the following four thermophilous herb associations. Three of them are locally not differentiated and represent single subassociations, whereas another one is differentiated into three subassociations:

Ass. Peucedanetum cervariae Kaiser 1926

Subass. Peucedanetum cervariae brachypodietosum pinnati Brzeg 2005

Ass. Campanulo bononiensis-Vicietum tenuifoliae Krausch in Th. Müller 1962

Subass. Campanulo bononiensis-Vicietum tenuifoliae typicum (Korneck 1974) Brzeg 2005

Ass. Geranio-Anemonetum sylvestris Th. Müller 1962

Subass. Geranio-Anemonetum sylvestris phleetosum phleoidis Brzeg et Wika 2011

Subass. G.-A.s. typicum (Korneck 1974) Brzeg 2002

Subass. G.-A.s. trifolietosum medii Th. Müller 1962 ex Brzeg et Wika 2011

Ass. Lathyro sylvestris-Vincetoxicetum hirundinariae (Hilbig 1971) Passarge 1979

Subass. Lathyro sylvestris-Vincetoxicetum hirundinariae typicum (Brzeg 2005) Brzeg et Wika 2011

\subsubsection{Peucedanetum cervariae}

(Table 1 - Tables 1-7 see on pages: $57-76$ )

Synonym: Sileno nutantis-Libanotidetum montanae Jeschke ex Passarge 1979; for more see Brzeg 2005

Occurrence conditions: In the "Murawy Dobromierskie" nature reserve, the Peucedanetum cervariae was the most widespread association of the Geranion sanguinei. It took mostly the form of the "post-terminal development stage" of xerothermic grasslands, covering relatively big areas. Its patches covered open spaces on hill summits and upper parts of slopes, the latter almost exclusively exposed to the south. On rendzina soils, phytocoenoses of this association demonstrated their exceptionally xerothermic character, compared to other communities of the mentioned alliance present in the object.

Physiognomy: Usually three vegetation layers were present. The shrub layer occurred almost always.
The herb layer was generally well-developed. It was represented by dicots, grasses and sedges. The cover of the moss layer was overally significant as well.

D ifferentiation. All phytocoenoses of the Peucedanetum cervariae found in the reserve represented the P.c. brachypodietosum pinnati subassociation, comprising the most calciphilous forms of the unit, though lacking any differentiating species.

\subsubsection{Campanulo bononiensis-Vicietum tenuifoliae} (Table 2)

Occurrence conditions: The association is related to partially altered habitats, such as shrub edges on ex-arable lands. In the reserve, phytocoenoses of this type occurred almost exclusively on sloping lands, mainly in lower parts of slopes (due to a better development of soil levels), mostly along shrub edges on abandoned arable fields, or in clearings within the shrub formation.

Phys i o g n o m y: Usually, three vegetation layers were present. The shrub layer was well developed. The herb layer was lush, and consisted of dicots and grasses. The abundancy of the moss layer varied significantly.

D iffere ntiation: The Campanulo bononiensisVicietum tenuifoliae was locally poorly differentiated and was represented only by the C.b.-V.t. typicum subassociation - showing no distinctive traits and lacking differentiating species; the subassociation included the most xerothermic forms of the association.

\subsubsection{Geranio-Anemonetum sylvestris (Table 3)}

S y n o n y m s: Thalictro-Geranietum sanguinei Korneck 1974, Adonido-Thalictretum mini Passarge 1997 Not: Thalictro mini-Geranietum sanguinei Korneck 1974 sensu Dengler 2004

Occurrence conditions: In the reserve, patches of the Geranio-Anemonetum sylvestris occurred on flat, as well as on sloping areas of any exposition or inclination. Phytocoenoses found in the object generally preferred initial rendzina soils, with even higher concentration of skeleton material in their profile, compared to the substrates overgrown by e.g. the Peucedanetum cervariae. They could be typically encountered on shrub edges in upper parts of slopes, and in former limestone quarries.

Phys i og no m y: Phytocoenoses of this unit present in the reserve were characterized by a distinct threelayer structure. The shrub layer consisted of species commonly found in all studied communities. The herb layer varied widely in terms of coverage from relatively dispersed to thick and exuberant. The moss layer was always present and relatively strong.

Differentiation: In accordance with the division by Brzeg $(2002,2005)$, phytocoenoses of the 
Geranio-Anemonetum sylvestris in the area represented the calciphilous (basiphilous) sub-association group. It included three sub-associations, and each of them was present in the reserve:

a) G.-A.s. phleetosum phleoidis (Table 3, rel. 1-6) the most xerothermic sub-association, recorded by Brzeg \& Wika (2014) from Kraków-Częstochowa Upland; it prefers the substrate characterized by the least concentration of humus; in the reserve it could be encountered near hilltops and, after all, in limestone excavations;

b) G.-A.s. typicum (Table 3, rel. 7-11) - an average, xerothermic subassociation, floristically rich, but lacking its own differentiating species;

c) G.-A.s. trifolietosum medii (Table 3, rel. 12-16) - a mesophilous subassociation, distinguished by addition of species coming from the Trifolion medii alliance; as for the phytocoenoses found in the reserve, those mesophilous traits were not clearly marked. This sub-association occurred on forest edges, in spatial complexes with Peucedanetum cervariae and shrubs in the northeastern part of the object, and here and there even at limestone quarries.

\subsubsection{Lathyro sylvestris-Vincetoxicetum hirundinariae (Table 4)}

S y n o n y m s : Ass.-Gr. Vincetoxicetum-Säume (Kuhn 1937) Passarge 1979 nom. inval., Artemisio campestrisVincetoxicetum hirundinariae Dengler et Krebs 2003, Origano-Vincetoxicetum hirundinariae Kolbek et Petřiček 1979 ex M. Wojterska 2003; for others - see Brzeg 2005

Occurrence conditions: In the "Murawy Dobromierskie" reserve, phytocoenoses of this association covered slopes of moderate to high inclination, always exposed to the south, mainly in their upper parts. Rendzina soils generally similar to those overgrown by phytocoenoses of Peucedanetum cervariae were the preferred substrate.

P hy s i o g n o m y: Patches of the Lathyro-Vincetoxicetum hirundinariae in the reserve were generally composed of three vegetation layers. However, the shrub layer was usually restricted to single young individuals of taxa forming adjacent patches of Pruno-Ligustretum, resulting from the competitiveness of the association leading herb species - Vincetoxicum hirundinaria. The herb layer stood out for its abundance. The moss layer was important as well.

Differentiation: The unit constituted the central association of the Geranion sanguinei. In the reserve, only one subassociation was found - L.s.-V.h. typicum. Phytocoenoses representing this unit showed lack of some xerothermic species, which are defined as differentiating for the subassociation (Agrimonia eupatoria, Origanum vulgare, Plantago media, Sca- biosa ochroleuca, and Veronica teucrium, see Brzeg \& Wika 2014).

\subsection{Vegetation changes between 1993-2012}

In the present analysis, two sets of relevés, diagnosed as: 1) the Thalictro-Salvietum pratensis (recorded in 1993), and 2) four communities of the Geranion sanguinei (documented in 2012), were compared with regard to floristic changes that accumulated over 19 years.

In the synoptic table (Table 6), floristic compositions of each association characterized in chapter 3.1. (columns 1-4), the species combination of these associations taken collectively as the Geranion sanguinei (column 5), and one of the above mentioned ThalictroSalvietum phytocoenoses, were put together. The table shows not only the current differentiation of xerothermic forb fringe communities, but also it enables to analyze, from syndynamical and species composition-related viewpoint, changes that non-forest vegetation of the reserve were subjected to during the period 1993-2012. Among character taxa of the four Geranion sanguinei associations, no distinct changes were noted. The share of Cirsio-Brachypodion pinnati character species, on the other hand, dropped significantly (e.g. Aster amellus, Seseli annuum, Campanula sibirica). The role of the Geranion sanguinei alliance plants remained at a comparable level, except for Anthericum ramosum which declined considerably in the course of that period. Appearance of some important species of this group: Campanula rapunculoides, Trifolium rubens, and Viola collina is also noteworthy. A substantial decrease in the share of mesophilous "meadow" grassland species of the Brometalia erecti can be noted.

Amidst the Festuco-Brometea taxa, notable changes can be stated. Excluding the inexplicably abundant occurrence of the newly noted moss species Homalothecium lutescens, majority of plants comprising the group in 1993 declined significantly (i.e. Anthemis tinctoria, Anthyllis vulneraria, Centaurea stoebe, Scabiosa ochroleuca, Stachys recta, Verbascum lychnitis) or even retreated (i.e. Acinos arvensis and Poa compressa). The emergence of a few taxa diagnostic for the TrifolioGeranietea sanguinei (e.g. Agrimonia eupatoria, Astragalus glycyphyllos, Chamaecytisus ruthenicus, Origanum vulgare, and Rubus caesius var. arvalis) should also be considered an important phenomenon. As for the Rhamno-Prunetea group, a prominent role of three shrub species in the composition of present communities (Cornus sanguinea, Ligustrum vulgare, and newly recorded Rosa dumalis) should be emphasized. Distinct changes in the species combination could also be observed within other syntaxonomical groups (the Molinio-Arrhenatheretea, the Koelerio-Corynephoretea, and the Artemisietea vulgaris), as well as within further accompanying taxa. 
The secondary succession process manifested itself most strikingly in the vegetation structure alteration, namely, a massive development of the moss layer, which attained in 2012 a mean cover of ca. 54\%, and was almost non-existent back in 1993. Simultaneously, the herb layer reasonably declined in cover, from $100 \%$ to ca. $80 \%$.

Using selected numerical parameters, an attempt was made to verify tendencies indicated in Table 6 . Table 7 shows that in the period 1993-2012, for 7 out of 11 syntaxonomic distinguished groups, an unambiguous decrease in the values of the above-mentioned indices took place. These groups constituted character taxa of the following units: Artemisietea vulgaris and Geranion sanguinei (with the values of $\mathrm{G}$ and $\mathrm{D}$ having sizeably dropped during that period), Trifolio-Geranietea sanguinei, Cirsio-Brachypodion pinnati, and FestucoBrometea (with the C value substantially higher for 1993 vs. 2012), Brometalia erecti, Koelerio-Corynephoretea, and the group of other accompanying taxa (with the values of all variables considerably higher for 1993). For character taxa of only two units, i.e.: MolinioArrhenatheretea and Rhamno-Prunetea, the value of any parameter notably increased over that time, i.e.: D; D and G, respectively. Within the sole group of characteristic taxa of the examined Geranion sanguinei associations, no significant changes were stated.

The data presented above show that the reserve vegetation underwent three major successional trends. These comprised: (1) retreat of thermophilous vegetation (manifested by a drop of values, estimated for the following groups of taxa: Brometalia erecti, CirsioBrachypodion, Festuco-Brometea, Geranion sanguinei, Koelerio-Corynephoretea, and Trifolio-Geranietea), (2) its replacement by a xerothermic shrub formation (demonstrated by $\mathrm{G}$ and $\mathrm{D}$ coefficients growth, estimated for the Rhamno-Prunetea taxa), and (3) expansion of the moss layer against a decline of the herb layer.

\section{Discussion}

As it was mentioned earlier, the recession of calcareous grasslands resulted from gradual abandonment of pastures, meadows, and arable fields which led to increased litter deposition, moistening and shading habitats, and, consequently, colonization of grasslands by shrubs and tall dicotyledonous herbs (Perzanowska \& Kujawa-Pawlaczyk 2004). This trend of changes was observed for a long time in many places of Central Europe (Kozłowska 1925; Kaiser 1926; Bornkamm \& Eber 1967; Głazek 1968, 1987; Faliński 1972; Dierschke 1974, 1985, 2006; Korneck 1974; Świerczyńska 1990; Wilmanns \& Sendtko 1995; Zerbe \& Schacht 1998; Willems 2001; Poschlod \& WallisDe Vries 2002; Bąba 2003; Babczyńska-Sendek 2005; Chytrý 2007;
Hegedüšová Vantarová \& Škodová 2014). However, the issue of the role of Trifolio-Geranietea sanguinei communities in this process has been rarely discussed so far (Bornkamm \& Eber 1967; Dierschke 1974, 1985, 2006; Korneck 1974; Brzeg 2005; Brzeg \& Wika 2014).

The studies on the "Murawy Dobromierskie" reserve vegetation (Szygendowski 2013) provided useful materials for analyzing this problem. It seems that xerothermic grasslands present there still in 1990s (Olaczek et al. 1993) underwent an advanced secondary succession. The documented grassland phytocoenoses were characterized by a high share of shrubs and xerothermic herbs; what is more, they were given a controversial syntaxonomical status. Their classification as the Thalictro-Salvietum pratensis seems dubious due to the lack of character species of this association (see Matuszkiewicz 2001; Ratyńska et al. 2010). It was related rather to the Origano-Brachypodietum vincetoxicetosum Medw.-Korn. et Kornaś 1963 or, some patches with Aster amellus, even to the Inuletum ensifoliae (Wnuk \& Pisarek 2008). The authors' observations from 2012 proved that a single patch of grassland of such type was still present in the reserve, and was located in the area where special protection measures impeding secondary succession were applied (i.e. repetive shrub cutting and disturbing of soil surface).

Regarding the results of the analysis presented above, it can be assumed that phytocoenoses of thermophilous herbs were widespread in the reserve back in 1993. However, the typical area of the relevés taken at that time (Olaczek et al. 1993) was nearly $100 \mathrm{~m}^{2}$, so that the vegetation mosaic, including calcareous grasslands, shrubbery tufts, and tall herb phytocoenoses, could have been incorporated. This can explain unexpectedly high values of indices, estimated for the Geranion sanguinei, the Rhamno-Prunetea, and the Trifolio-Geranietea sanguinei, based on the material from 1993 (Table 7). Moreover, as stated previously, two dominant moss taxa were not included in the analysis of the parameters. Taking this into account, the above-mentioned phenomenon of a rapid development of the moss layer in the period 1993-2012 could have contributed to the underestimation of all values, especially of the cover index, for the latter year. Bearing this in mind, it seems that a direct comparison of full floristic compositions of communies in a synoptic table would give a better illustration of changes than using numerical parameters, estimated on the basis of partly different groups of taxa, quality-wise.

In the face of rapidly ongoing processes of secondary succession as well as apparent ineffectiveness of undertaken protection measures, the reserve protection plan needs urgent revision. This also applies to other "steppetype" nature reserves, whose vegetation was not been studied for decades. Firstly, the most precise recognition 
of the area vegetation is needed, based on the current syntaxonomic division by Brzeg (2005), Ratyńska et. al. (2010), and Brzeg \& Wika (2014), so that associations of the Trifolio-Geranietea sanguinei class are not missed anymore. Secondly, the major protection goal of the plan should also be reconsidered. Namely, thermophilous herb communities and forest ecosystems, as a refuge of rare and protected flora, deserve protection as much as calcareous grasslands and xerothermic shrubs do. Last but not least, considering such low effectiveness of protection measures so far implemented in the reserve, i.e. shrub cutting and grassland mowing, we stipulate their partial cessation. Instead, we propose rehabilitation of traditional methods of land use, such as grazing and controlled burning. Such diversification of protection activities would obviously increase implementation costs of the plan, but this is crucial for impeding the expansion of shrubs, and preserving the vegetation mosaic of forests, xerothermic shrubs, thermophilous herbs, and calcareous grasslands.

Acknowledgements. We are grateful to Professor Maria Wojterska for her comments. We would also like to thank the staff of Przedbórz Landscape Park for making valuable sources of information available for us.

\section{References}

BabczyńsKa-Sendek B. 2005. Problemy fitogeograficzne i syntaksonomiczne kserotermów Wyżyny Śląskiej. 237 pp. Wyd. Uniw. Śląskiego, Katowice.

BĄBA W. 2003. Ekologiczne podstawy ochrony aktywnej i kształtowania ekosystemów muraw kserotermicznych w Ojcowskim Parku Narodowym i otulinie. II. Zmiany składu florystycznego badanych poletek. Prądnik. Prace Muzeum Szafera 13: 77-94.

Bobbink R. \& Willems J. H. 1987. Increasing dominance of Brachypodium pinnatum (L.) Beauv. in chalk grasslands: a threat to a species-rich ecosystem. Biol. Conserv. 40: 301-314.

BobBink R. \& Willems J. H. 1993. Restoration management of abandoned chalk grassland in the Netherlands. Biodivers. Conserv. 2: 616-626.

Bornkamm R. \& Eber W. 1967. Die Pflanzengesellschaften der Keuperhügel bei Friedland (Kr. Göttingen). Schriftenreihe für Vegetationskunde 2: 135-160.

Braun-Blanquet J. 1964. Pflanzensoziologie. 3. Aufl. 865 pp. Springer-Verlag., Wien-New York.

Brzeg A. 2002. Występowanie, zróżnicowanie i specyfika regionalna zespołu Geranio-Anemonetum sylvestris Th. Müller 1962 w Wielkopolsce. Bad. Fizjogr. Pol. Zach., B-Botanika 51: 55-81.

BRzEG A. 2005. Zespoły kserotermofilnych ziołorośli okrajkowych z klasy Trifolio-Geranietea sanguinei Th. Müller 1962 w Polsce. 236 pp. Bogucki Wyd. Naukowe, Poznań.

BRzeg A. \& WiKA S. 2014. Termofilne ziołorośla okrajkowe z klasy Trifolio-Geranietea sanguinei Th. Müller 1962 na obszarze środkowej części Wyżyny Krakowsko-Częstochowskiej (wyd. II poprawione). 179 pp. Zespół Parków Krajobrazowych Województwa Śląskiego, Katowice.

ChytrÝ M. (ed.). 2007. Vegetace České republiky. 1. Travinná a keříčková vegetace. 526 pp. Academia, Praha.

DierschKe H. 1974. Saumgesellschaften im Vegetations- und Standortsgefälle an Waldrändern. Scripta Geobot. 6, 246 pp.. Verl. E. Goltze KG, Göttingen.
DiERSCHKE H. 1985. Experimentelle Untersuchungen zur Bestandesdynamik von Kalkmagerrasen (Mesobromion) in Südniedersachsen. 1. Vegetationsentwicklung auf Dauerflächen 1972-1984. Münstersche Geogr. Arb. 20: 9-24.

Dierschke H. 2006. Sekundär-progressive Sukzession eines aufgelassenen Kalkmagerrasens. Dauerflächenuntersuchungen 1987-2002. Hercynia 39: 223-245.

Dzwonko Z. 2007. Przewodnik do badań fitosocjologicznych. In: Vademecum Geobotanicum, 304 pp. Sorus, Instytut Botaniki Uniwersytetu Jagiellońskiego, Poznań-Kraków.

Ellenberg H. 1996. Vegetation Mitteleuropas mit den Alpen in ökologischer, dynamischer und historischer Sicht. 5th ed. 1095 pp. Ulmer, Stuttgart.

Enyedi Z. M., Ruprecht E. \& DeÁk M. 2008. Long-term effects of the abandonment of grazing on steppe-like grasslands. Appl. Veg. Sci. 11: 55-62.

FALIŃSKI J. B. 1972. Antropogeniczne zagrożenia i program ochrony muraw kserotermicznych na kemach w północnej części Równiny Bielskiej. Phytocoenosis 4(1): 287-305.

GŁAzeK T. 1968. Roślinność kserotermiczna Wyżyny Sandomierskiej i Przedgórza Iłżeckiego. Monogr. Bot. 25: 1-135.

GŁAzEK T. 1987. Murawy i zarośla kserotermiczne wzgórz wapiennych Okręgu Chęcińskiego. 40 pp. Wyd. Geologiczne, Warszawa.

GroDZIŃSKA K. 1970. Zbiorowiska kserotermiczne Skalic Nowotarskich i Spiskich (Pieniński Pas Skałkowy). Fragm. Flor. Geobot. 3(16): 401-432.

Hahn A., Andres C. \& Becker T. 2012. Veränderungen der Steppenrasen des NSG "Badraer Lehde-Großer Eller" im Kyffhäusergebirge (Thüringen) zwischen 1993 und 2012. Nuuk Ecological Research Operations 6: 101-115.

HegedüŠová VANTAROvÁ K. \& ŠKodová I. (eds.). 2014. Rastlinné spoločenstvá Slovenska. 5. Travinno-bylinná vegetácia. $581 \mathrm{pp}$. Veda, Bratislava.

Kaiser E. 1926. Die Pflanzenwelt des Hennebergisch-Fränkischen Muschelkalkgebietes. 280 pp. Rep. spec. nov. reg. veg., Beih., 44, Dahlem bei Berlin. 
Kondracki J. 1998. Geografia regionalna Polski. 441 pp. Wyd. Nauk. PWN, Warszawa.

KoRNECK D. 1974. Xerothermvegetation in Rheinland-Pfalz und Nachbargebieten. 198 pp. Schriftenreihe Vegetationskunde 7., Bonn-Bad Godesberg.

KozŁowska A. 1925. Zmienność kostrzewy owczej (Festuca ovina L.) w związku z sukcesją zespołów stepowych na Wyżynie Małopolskiej. PAU, Spraw. Kom. Fizjogr. 58/59: 63-110.

Kratochwil A. 1984. Pflanzengesellschaften und Blütenbesucher-Gemeinschaften: biozönologische Untersuchungen in einem nicht mehr bewirtschafteten Halbtrockenrasen (Mesobrometum) im Kaiserstuhl (Südwestdeutschland). Phytocoenologia 11: 455669.

Kupferschmid A. D., Stampfli A. \& Newbery D. M. 2000. Dispersal and Microsite Limitation in an Abandoned Calcareous Grassland of the Southern Prealps. Folia Geobot. 35: 125-141.

Matuszkiewicz W. 2001. Przewodnik do oznaczania zbiorowisk roślinnych Polski. In: J. B. FALIŃSKi (ed.). Vademecum Geobotanicum 3, 537 pp. Wyd. Nauk. PWN, Warszawa.

Medwecka-Kornaś A. \& Kornaś J. 1963. Mapa zbiorowisk roślinnych Ojcowskiego Parku Narodowego. Ochr. Przyr. 29: 17-87.

Medwecka-Kornaś A. \& Kornaś J. 1972. G. Zespoły stepów i suchych muraw. In: W. SZAFER \& K. ZARZYCKI (eds.). Szata roślinna Polski, 1, pp. 352-366. PWN Warszawa.

MüLler TH. 1962. Die Saumgesellschaften der Klasse Trifolio-Geranietea sanguinei. Mitt. Flor-soz. Arbeitsgem., N.F. 9: 95-140.

MüLlER TH. 1978. Klasse: Trifolio-Geranietea sanguinei Th. Müller 61. Helio-thermophile Saumgesellschaften, Staudenhalden, Laubwiesen. In: E. OBERDORFER (ed.). Süddeutsche Pflanzgesellschaften. II. Pflanzensoziologie 10: 240-298. G. Fischer Verl., Jena.

Ochyra R., Żarnowiec J. \& Bednarek-Ochyra H. 2003. Census Catalogue of Polish Mosses. In: Z. Mirek (ed.). Biodiversity of Poland, 3, $372 \mathrm{pp}$. Polish Academy of Sciences, Institute of Botany, Kraków.

Olaczek R., Kurzac M., BabsKa D., Wnuk Z., ZaŁuski W. \& FrAnCZAK E. 1993. Rezerwat stepowy Murawy Dobromierskie. Plan ochrony na lata 1994-2013. Urząd Wojewódzki w Piotrkowie Trybunalskim, Łódź-Piotrków Trybunalski.

PAWŁowsKi B. 1972. Skład i budowa zbiorowisk roślinnych oraz metody ich badania. In: W. SZAFER \& K. ZARZYCKI (eds.). 1959. Szata roślinna Polski, 1, pp. 237-269. PWN Warszawa.

Perzanowska J. \& Kujawa-Pawlaczyk J. 2004. Murawy kserotermiczne. In: J. Herbich (ed.). Murawy, łąki, ziołorośla, wrzosowiska, zarośla. Poradniki ochrony siedlisk i gatunków Natura 2000 - podręcznik meto- dyczny. 3, pp. 117-139. Ministerstwo Środowiska, Warszawa.

Poschlod P. \& WallisDe VRies M. F. 2002. The historical and socio-economic perspective of calcareous grasslands. Lessons learnt from the distant and recent past. Biol. Conserv. 104: 361-376.

RatyŃSKa H., Wojterska M., Brzeg A. \& KoŁacz M. 2010. Multimedialna encyklopedia zbiorowisk roślinnych Polski ver. 1.1. Uniw. im. Kazimierza Wielkiego, Inst. Eduk. Tech. Inf., Bydgoszcz-Poznań-Warszawa.

Regulation 2014. Regulation of the Minister of the Environment of 9 October 2014 on plant species protection. Journal of Laws 2014 item 1409.

Rusina S. \& KiehL K. 2010. Long-term changes in species diversity in abandoned calcareous grasslands in Latvia. Tuexenia 30: 467-486.

RutKowski L. 2004. Klucz do oznaczania roślin naczyniowych Polski niżowej. Wyd. II, popr. i unowocześnione, 814 pp. Wyd. Nauk. PWN, Warszawa.

Schrautzer J., Jansen D., Breuer M. \& Nelle O. 2009. Succession and management of calcareous dry grasslands in the Northern Franconian Jura, Germany. Tuexenia 29: 339-351.

SŁawnikowski O. 1998. ${ }^{\circ}$ ExGrad for Windows 1.01. Poznań.

SzYGENDOwSKI T. 2013. Nowe zbiorowiska roślinności kserotermicznej w rezerwacie "Murawy Dobromierskie" (Wyżyna Małopolska). Master's thesis, Department of Plant Ecology and Environmental Protection, Adam Mickiewicz University, Poznań, Poland.

ŚwIERCZYŃSKA S. 1990. Problem zachowania zbiorowisk stepowych na podstawie badań prowadzonych na Lubelszczyźnie. Prądnik. Prace Muzeum Szafera 2: 29-34.

Willems J. H. 2001. Problems, approaches and results in restoration of Dutch calcareous grasslands during the last 30 years. Restoration Ecolology 9: 147-154.

Wilmanns O. \& KRAtochwil A. 1983. Naturschutz-bezogene Grundlagen-Untersuchungen im Kaiserstuhl. Veröff. Natursch. Landschaftspfl. Baden-Württ. Beiheft 34: 39-56.

Wilmanns O. \& SendtKo A. 1995. Sukzessionslinien in Kalkmagerrasen unter besonderer Berücksichtigung der Schwäbischen Alb. Veröff. Natursch. Landschaftspfl. Baden-Württ. Beiheft 83: 257-282.

WNuK Z. 1981. Goryczka orzęsiona, zawilec wielkokwiatowy i dziewięćsił bezłodygowy w Paśmie PrzedborskoMałogoskim. Chrońmy Przyr. Ojcz. 37(5): 58-66.

WNuk Z. 1984. Gentiana ciliata L. w Paśmie PrzedborskoMałogoskim. Acta Univ. Lodz., Folia bot. 2: 31-39.

Wnuk Z. \& PisareK W. 2008. Flora. In: Z. WNUK (ed.). Przedborski Park Krajobrazowy. 20 lat istnienia PPK (1988-2008), pp. 55-126. Wyd. Uniw. Rzeszowskiego, Rzeszów.

Zerbe S. \& Schacht T. 1998. Vegetation and successional stages in chalk quarries on Jasmund (Rügen Island, NE Germany). Fragm. Flor. Geobot. 43(1): 117-146. 


\section{Tables}


Table 1. Floristic composition of Peucedanetum cervariae

\begin{tabular}{|c|c|c|c|c|c|c|c|c|c|c|c|c|}
\hline \multicolumn{2}{|c|}{ Successive No. } & 1 & 2 & 3 & 4 & 5 & 6 & 7 & 8 & 9 & 10 & \multirow{11}{*}{ 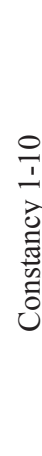 } \\
\hline \multicolumn{2}{|c|}{ Field No. } & 48 & 4 & 1 & 7 & 44 & 47 & 45 & 46 & 43 & 42 & \\
\hline \multirow{2}{*}{ Date (2012) } & \multirow{2}{*}{$\begin{array}{l}\text { day } \\
\text { month }\end{array}$} & 16 & 2 & 2 & 2 & 16 & 16 & 16 & 16 & 10 & 10 & \\
\hline & & VIII & VI & VI & VI & VIII & VIII & VIII & VIII & VIII & VIII & \\
\hline \multicolumn{2}{|c|}{ Slope exposure } & SWW & $\mathrm{E}$ & SW & SEE & SSW & - & $\mathrm{S}$ & SW & SSW & SSW & \\
\hline \multicolumn{2}{|c|}{ Inclination $\left[^{\circ}\right]$} & 7 & 5 & 7 & 7 & 10 & - & 8 & 7 & 10 & 7 & \\
\hline \multicolumn{2}{|c|}{ Density of shrub layer [\%] } & $<5$ & 10 & 12 & 10 & 5 & + & 25 & 10 & 5 & 15 & \\
\hline \multicolumn{2}{|c|}{ Cover of herb layer [\%] } & 75 & 90 & 85 & 30 & 85 & 95 & 85 & 90 & 70 & 80 & \\
\hline \multicolumn{2}{|c|}{ Cover of moss layer [\%] } & 50 & 7 & 10 & 98 & 65 & 40 & 65 & 75 & 75 & 60 & \\
\hline \multicolumn{2}{|c|}{ Area of relevé $\left[\mathrm{m}^{2}\right]$} & 30 & 20 & 15 & 15 & 50 & 20 & 20 & 20 & 25 & 25 & \\
\hline \multicolumn{2}{|c|}{ Number of taxa } & 42 & 62 & 45 & 51 & 43 & 40 & 37 & 33 & 41 & 44 & \\
\hline
\end{tabular}

I. Ch., *D. Ass.

Peucedanum cervaria

$\begin{array}{ccccccccccc}2.1 & 3.4 & 3.1 & 4.4 & 2.1 & 3.3 & 3.3 & 3.3 & 3.2 & 4.3 & \text { V } \\ +.2 & +.2 & . & 1.2 & . & . & . & . & . & +^{\circ} & \text { II }\end{array}$

*Aster amellus (C-B)

$\begin{array}{llll}+.2 & +.2 \quad & .2\end{array}$

II. Ch., *D. Geranion sanguinei

*Galium boreale (M-A)

$\begin{array}{llllllllll}+ & 2.1 & 1.2 & 2.1 & 1.1 & + & \mathrm{r} & + & + & +\end{array}$

Vincetoxicum hirundinaria

Anemone sylvestris

Anthericum ramosum

3.3

Thalictrum minus

Vicia tenuifolia

Viola collina

Geranium sanguineum

Primula veris

$$
\begin{array}{cccccccccc}
3.4 & 1.2 & + & 1.2 & 1.2 & 1.1 & . & + & + & \mathrm{V} \\
1.2 & 1.2 & +.2 & +.2 & 2.2 & . & . & . & . & \text { III } \\
. & + & \mathrm{r} & 1.3 & . & . & . & . & . & \mathrm{II} \\
2.2 & 1.1 & + & . & . & . & . & . & . & \mathrm{II} \\
. & . & . & +.2 & \mathrm{r}^{\mathrm{0}} & +{ }^{\circ} & \mathrm{r}^{\mathrm{0}} & . & . & \mathrm{II} \\
. & . & +.2 & . & +.2 & + & +.2 & . & . & \mathrm{II} \\
. & . & . & + & . & . & \mathrm{r} & . & . & \mathrm{II} \\
. & 2.2 & 2.2 & . & . & . & . & . & . & \mathrm{I} \\
+.2 & 2.2 & . & . & . & . & . & . & . & \mathrm{I}
\end{array}
$$$$
\begin{array}{lll}
1.1 & + & + \\
&
\end{array}
$$$$
\begin{array}{cccccccccc}
\cdot & + & \mathrm{r} & 1.3 & \cdot & \cdot & \cdot & \cdot & \cdot & \text { II } \\
2.2 & 1.1 & + & & & & & & &
\end{array}
$$

III. Ch., *D. Origanetalia, Trifolio-Geranietea

Poa angustifolia

Agrimonia eupatoria

Viola hirta

Knautia arvensis fo.

Agrimonia procera

Astragalus glycyphyllos

Hypericum perforatum

Coronilla varia

Clinopodium vulgare

Galium album fo.

Chamaecytisus ruthenicus

*Rubus caesius var. arvalis (A)

Origanum vulgare

\section{Ch. Festuco-Brometea}

Euphorbia cyparissias

Abietinella abietina d

Homalothecium lutescens $\mathrm{d}$

Sanguisorba minor

Seseli annuum (C-B)

Medicago falcata

Potentilla arenaria

Carex caryophyllea

Veronica spicata

Scabiosa ochroleuca

Salvia pratensis

Achillea collina

Anthyllis vulneraria

Carlina vulgaris (B)

Melampyrum arvense (C-B)

+.2
+
+
.
.
+
+
+
.
.
.
.
.
.

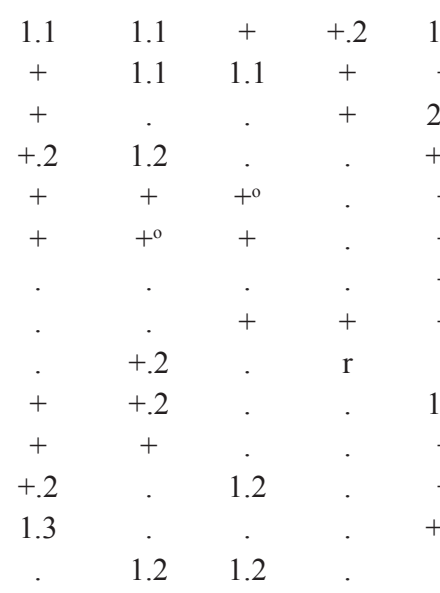$$
\begin{array}{cccccc}
1.2 & 1.1 & 1.1 & + & 1.1 & \mathrm{~V} \\
+ & + & + & + & + & \mathrm{V} \\
2.1 & + & 1.1 & + & + & \mathrm{IV} \\
+.2 & +.2 & \mathrm{r} & + & +.2 & \mathrm{IV} \\
+ & + & + & . & + & \mathrm{IV} \\
+ & \mathrm{r} & + & . & . & \mathrm{IV} \\
+ & + & + & + & + & \mathrm{III} \\
+ & + & . & + & . & \mathrm{III} \\
. & + & \cdot & + & + & \mathrm{III} \\
1.2 & . & \cdot & + & . & \mathrm{II} \\
+ & \cdot & \cdot & \mathrm{r} & . & \mathrm{II} \\
+ & \cdot & . & . & . & \mathrm{II} \\
+.2 & +.2 & \cdot & \cdot & . & \mathrm{II} \\
. & . & . & . & . & \mathrm{I}
\end{array}
$$

1.1

$\begin{array}{cccccccccc}+ & +^{\circ} & + & 1.3 & + & + & 1.1 & 1.2 & 1.1 & \mathrm{~V} \\ 1.2 & +.2 & 3.3 & 3.3 & 3.3 & +.2 & 2.2 & 4.4 & 3.3 & \mathrm{~V} \\ 1.2 & 1.2 & +.2 & 3.3 & 2.2 & 4.4 & 3.3 & +.2 & 3.3 & \mathrm{~V} \\ + & \mathrm{r} & + & + & + & + & + & + & + & \mathrm{V} \\ +^{\circ} & \mathrm{r}^{\circ} & . & + & + & + & . & + & + & \mathrm{IV} \\ + & +.2 & 1.2 & 1.1 & . & . & 1.1 & 1.2 & 1.1 & \mathrm{IV} \\ . & . & +.2 & 2.2 & +.2 & + & +.2 & 1.2 & 1.2 & \mathrm{IV} \\ . & . & + & +.2 & 1.1 & 1.1 & . & 1.2 & 1.1 & \mathrm{IV} \\ + & . & . & 1.2 & +.2 & +.2 & . & 1.2 & 1.2 & \mathrm{IV} \\ . & . & . & + & \mathrm{r} & + & \mathrm{r} & + & + & \mathrm{IV} \\ . & . & +.2 & 1.1 & + & 2.1 & 1.1 & 2.1 & 2.1 & \mathrm{IV} \\ + & . & + & . & + & . & + & + & 1.1 & \mathrm{III} \\ . & . & + & . & . & . & . & + & + & \mathrm{II} \\ . & + & + & \mathrm{r} & . & . & . & . & + & \mathrm{II} \\ . & . & . & \mathrm{r} & \mathrm{r} & . & . & + & + & \mathrm{II}\end{array}$


Polygala comosa (B)

Thymus pulegioides (B)

Carex flacca (B)

Achillea pannonica

Salvia verticillata (C-B)

Centaurea scabiosa

Brachypodium pinnatum (B)

Centaurea stoebe

Allium oleraceum

Petrorhagia prolifera

\section{Ch. Molinio-Arrhenatheretea}

Arrhenatherum elatius

Dactylis glomerata

Plantago lanceolata

Veronica chamaedrys

Briza media

Carex hirta

Achillea millefolium

Leontodon hispidus s.s.

Stachys officinalis

\begin{tabular}{|c|c|c|c|c|c|c|c|c|c|}
\hline . & +.2 & . & +.2 & . & . & . & $\mathrm{r}$ & . & + \\
\hline+.2 & +.2 & . & +.2 & . & +.2 & . & . & . & . \\
\hline . & 1.2 & . & +.2 & . & . & . & . & . & +.2 \\
\hline+ & . & . & . & + & . & + & . & . & . \\
\hline . & . & . & . & . & . & $\mathrm{r}$ & + & . & $\mathrm{r}$ \\
\hline . & . & . & . & . & . & + & 1.1 & . & 1.1 \\
\hline . & . & 4.4 & . & 3.3 & . & . & . & . & . \\
\hline $\mathrm{r}$ & . & . & . & +.2 & . & . & . & . & . \\
\hline . & . & . & . & . & . & + & + & . & . \\
\hline $\mathrm{r}$ & . & . & . & . & . & $r$ & . & . & . \\
\hline
\end{tabular}

VI. Ch. Artemisietea vulgaris

Medicago lupulina

\begin{tabular}{|c|c|c|c|c|c|c|c|c|}
\hline 1.2 & . & 1.1 & 1.1 & 1.1 & 1.1 & + & 2.1 & + \\
\hline+ & + & . & + & +.2 & . & . & +.2 & +.2 \\
\hline . & . & + & . & + & +.2 & . & + & + \\
\hline $\mathrm{r}^{\mathrm{o}}$ & $\mathrm{r}$ & . & . & + & . & + & . & . \\
\hline 1.2 & . & 2.2 & . & . & . & . & 3.2 & . \\
\hline . & + & . & . & . & . & . & + & +.2 \\
\hline . & . & $\mathrm{r}$ & $\mathrm{r}$ & . & . & + & . & . \\
\hline . & . & + & . & . & . & . & . & $\mathrm{r}$ \\
\hline . & . & . & . & . & . & . & . & . \\
\hline
\end{tabular}

\section{Ch. Koelerio-Corynephoretea}

Sedum acre

\section{Ch. Rhamno-Prunetea}

Prunus spinosa $\mathrm{b} / \mathrm{c}$

Cornus sanguinea $\mathrm{b} / \mathrm{c}$

Ligustrum vulgare $\mathrm{b} / \mathrm{c}$

Rhamnus catharticus b/c

Rosa canina b/c

Rosa dumalis b/c

\section{Ch. Querco-Fagetea}

Carex montana

Melica nutans

\section{Ch. Vaccinio-Piceetea}

Juniperus communis $\mathrm{b} / \mathrm{c}$

Pinus sylvestris $\mathrm{b} / \mathrm{c}$

\section{Others}

Hieracium pilosella

Linum catharticum

Cruciata glabra

Quercus robur b/c

Convolvulus arvensis

Pteridium aquilinum

Plagiomnium affine $\mathrm{d}$

Thuidium philibertii $\mathrm{d}$

$\cdot$
+
+
$\cdot$
+
+

$\begin{array}{cc}+ & + \\ + & 2.1 \\ 1.2 & 1.2 \\ + & + \\ 1.1 & + \\ \mathrm{r} & \end{array}$

$\begin{array}{ccccccccc}+ & + & 1.1 & + & 2.1 & 2.1 & 1.1 & 2.1 & \mathrm{~V} \\ 2.1 & 1.2 & . & . & + & + & . & + & \text { IV } \\ 1.2 & 1.2 & +.2 & . & . & +.2 & . & + & \text { IV } \\ + & . & + & + & + & . & . & . & \text { III } \\ +.2 & + & + & . & . & . & . & . & \text { II } \\ . & . & . & + & . & + & . & . & \text { II }\end{array}$

$$
\begin{array}{lllll}
. & \cdot & \cdot & \cdot & \mathrm{I} \\
. & . & . & . & \mathrm{I}
\end{array}
$$

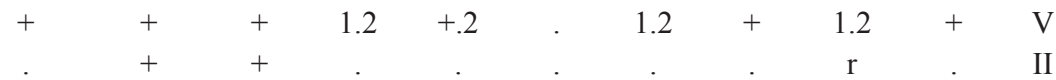

Sporadic taxa: II. Trifolium alpestre 2(+); Trifolium rubens 2(+.2); III. Fragaria vesca 2(+.2); Senecio jacobaea fo. 9(r); IV. Alchemilla glaucescens (B) 5(+); Arabis hirsuta 3(r); Orobanche caryophyllacea 4(r); Phleum phleoides 4(+.2); Pimpinella saxifraga 3(r); Plantago media (B) 2(+); Ranunculus bulbosus (B) $2(+)$; Stachys recta 2(+); Thymus pannonicus ssp. marschallianus 6(1.2); V. Centaurea jacea 5(+); Festuca rubra 10(+); Leontodon hispidus ssp. danubialis 2(+); Lotus corniculatus 4(+); Trifolium pratense 1(+.2); Vicia cracca 3(+); VI. Elymus repens 2(r); Falcaria vulgaris 10(r); Picris hieracioides 9(+); VII. Sedum sexangulare 4(+.2); VIII. Crataegus monogyna b/c 9(+); Frangula alnus b/c 2(r); Populus tremula b/c 2(+); IX. Euonymus verrucosus b/c 3(+); Fagus sylvatica b/c 2(r); X. Pleurozium schreberi d 2(+.2); Pseudoscleropodium purum d 2(+.2); Rubus saxatilis 3(1.2); XI. Agrostis capillaris 4(+.2); Brachythecium rutabulum d 5(+.2); Cimicifuga europaea 2(+.2); Fissidens cristatus d 2(+.2); 4(+.2); Silene vulgaris 1(+); Tragopogon sp. 4(r) 
Table 2. Floristic composition of Campanulo bononiensis-Vicietum tenuifoliae

\begin{tabular}{|c|c|c|c|c|c|c|c|c|c|c|c|c|c|}
\hline Successive No. & 1 & 2 & 3 & 4 & 5 & 6 & 7 & 8 & 9 & 10 & 11 & 12 & \\
\hline Field No. & 14 & 13 & 17 & 15 & 6 & 2 & 39 & 40 & 41 & 22 & 37 & 38 & \\
\hline \multirow{2}{*}{$\begin{array}{ll}\text { Date (2012) } & \text { day } \\
\text { month }\end{array}$} & 3 & 3 & 3 & 2 & 2 & 29 & 29 & 29 & 14 & 29 & 29 & 3 & $\stackrel{1}{7}$ \\
\hline & VI & VI & VI & VI & VI & VII & VII & VII & VII & VII & VII & VI & $\frac{1}{2}$ \\
\hline Slope exposure & NW & $\mathrm{N}$ & SE & SE & SE & $\mathrm{S}$ & SSE & SSE & SSW & $\mathrm{N}$ & $\mathrm{S}$ & $\mathrm{S}$ & है \\
\hline Inclination $\left[^{\circ}\right]$ & 5 & 7 & 5 & 3 & 7 & 5 & 10 & 3 & 10 & 3 & 3 & 5 & 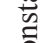 \\
\hline Density of shrub layer [\%] & 15 & 10 & 25 & 5 & 5 & 5 & 25 & 20 & 10 & 5 & + & 30 & ن \\
\hline Cover of herb layer [\%] & 80 & 85 & 100 & 100 & 90 & 90 & 70 & 75 & 50 & 100 & 100 & 60 & \\
\hline Cover of moss layer [\%] & 80 & 60 & 5 & 10 & - & 70 & 50 & 70 & 80 & 20 & + & 40 & \\
\hline Area of relevé $\left[\mathrm{m}^{2}\right]$ & 15 & 15 & 25 & 10 & 20 & 8 & 15 & 25 & 20 & 20 & 25 & 20 & \\
\hline Number of taxa & 47 & 40 & 35 & 39 & 33 & 37 & 28 & 29 & 34 & 35 & 30 & 32 & \\
\hline \multicolumn{14}{|l|}{ I. Ch., *D. Ass. } \\
\hline Vicia tenuifolia & 4.4 & 3.4 & 5.4 & 5.5 & 3.4 & 3.4 & 3.3 & 3.2 & 2.2 & 4.3 & 3.3 & 3.3 & V \\
\hline *Pimpinella nigra $(\mathrm{F}-\mathrm{B})$ & 1.1 & 1.1 & + & . & + & $\cdot$ & . & $\cdot$ & $\cdot$ & . & . & . & II \\
\hline *Falcaria vulgaris (A) & . & + & 1.1 & $1.1^{\circ}$ & . & . & . & . & . & . & . & . & II \\
\hline Campanula bononiensis & . & . & $\cdot$ & + & . & . & . & . & . & . & . & . & + \\
\hline \multicolumn{14}{|l|}{ II. Ch., *D. Geranion sanguinei } \\
\hline Fragaria viridis & 3.1 & 3.4 & 2.1 & 2.1 & + & 2.1 & . & 2.1 & + & 2.1 & 2.1 & + & $\mathrm{V}$ \\
\hline *Galium boreale (M-A) & . & . & +.2 & 1.2 & . & $\mathrm{r}$ & . & + & +.2 & . & . & . & III \\
\hline Peucedanum cervaria & . & . & 1.3 & . & 1.1 & + & + & . & . & . & . & . & II \\
\hline Vincetoxicum hirundinaria & . & . & 1.2 & +.2 & 1.1 & . & . & . & . & . & . & . & II \\
\hline Primula veris & . & . & . & . & + & 2.1 & . & . & . & $\mathrm{r}$ & . & . & II \\
\hline Thalictrum minus & . & . & . & . & . & $\cdot$ & + & + & + & . & . & . & II \\
\hline Campanula rapunculoides & + & +.2 & . & . & . & . & . & . & . & . & . & . & I \\
\hline \multicolumn{14}{|c|}{ III. Ch. *D. Origanetalia, Trifolio-Geranietea } \\
\hline Poa angustifolia var. setacea & + & 1.1 & 1.1 & 1.1 & $+^{\circ}$ & + & 1.1 & 2.1 & . & 1.1 & + & + & $\mathrm{V}$ \\
\hline *Rubus caesius var. arvalis (A) & + & 1.2 & 2.1 & 2.1 & 1.1 & 3.4 & +.2 & +.2 & . & . & 4.4 & 1.2 & V \\
\hline Galium verum fo. & 2.1 & 2.1 & +.2 & +.2 & 1.1 & $\cdot$ & . & + & + & 1.1 & +.2 & + & $\mathrm{V}$ \\
\hline Agrimonia procera & + & 1.1 & $\mathrm{r}$ & + & $\mathrm{r}$ & . & + & + & . & + & $\mathrm{r}$ & . & IV \\
\hline Agrimonia eupatoria & + & . & . & . & . & + & + & + & + & 1.1 & + & + & IV \\
\hline Knautia arvensis fo. & + & $\mathrm{r}$ & . & & $\mathrm{r}^{\mathrm{o}}$ & . & . & $\mathrm{r}$ & + & + & + & + & IV \\
\hline Hypericum perforatum & . & . & +.2 & + & $+^{\circ}$ & + & $+^{\circ}$ & . & . & + & $\mathrm{r}$ & + & IV \\
\hline Viola hirta & . & . & + & . & + & 1.2 & + & . & . & . & . & + & III \\
\hline Astragalus glycyphyllos & . & . & . & . & +.2 & $\cdot$ & . & + & + & . & + & . & II \\
\hline Galium album fo. & + & . & . & . & + & $\cdot$ & . & . & . & + & . & . & II \\
\hline Origanum vulgare & . & +.2 & . & . & . & +.2 & . & . & . & . & . & . & I \\
\hline Coronilla varia & + & . & . & + & . & . & . & . & . & . & . & . & I \\
\hline \multicolumn{14}{|l|}{ IV. Ch. Festuco-Brometea } \\
\hline Homalothecium lutescens $\mathrm{d}$ & 2.2 & 3.3 & 1.2 & . & . & 4.4 & 3.4 & 4.4 & 4.4 & 2.2 & + & 3.4 & V \\
\hline Sanguisorba minor & 1.1 & + & + & + & . & . & + & + & + & + & + & + & $\mathrm{V}$ \\
\hline Achillea collina & + & + & $+^{o}$ & . & . & + & $\mathrm{r}^{\mathrm{o}}$ & $+^{\circ}$ & 1.1 & + & + & + & V \\
\hline Centaurea scabiosa & 1.1 & 2.1 & + & + & . & . & + & + & + & 1.1 & + & 1.1 & $\mathrm{~V}$ \\
\hline Euphorbia cyparissias & + & . & + & . & $+^{\circ}$ & . & $\mathrm{r}$ & + & + & + & . & + & IV \\
\hline Carex caryophyllea & . & +.2 & . & + & $+^{\circ}$ & . & +.2 & +.2 & 1.1 & . & +.2 & . & III \\
\hline Melampyrum arvense (C-B) & . & . & 1.2 & + & . & . & + & + & $\mathrm{r}$ & + & $\cdot$ & + & III \\
\hline Salvia pratensis & . & . & 1.2 & + & . & . & 1.1 & 1.1 & 1.1 & . & . & 1.1 & III \\
\hline Abietinella abietina $\mathrm{d}$ & 2.2 & 2.3 & . & . & . & . & 2.2 & 2.2 & 2.3 & . & . & $\cdot$ & III \\
\hline Medicago falcata & $\cdot$ & +.2 & +.2 & . & 1.1 & + & . & $\cdot$ & $\cdot$ & $\mathrm{r}$ & . & . & III \\
\hline Scabiosa ochroleuca & . & . & + & . & . & . & $\mathrm{r}^{\mathrm{o}}$ & $\mathrm{r}$ & + & . & + & . & III \\
\hline Carlina vulgaris (B) & + & + & . & + & . & . & . & . & + & . & + & . & III \\
\hline Salvia verticillata $(\mathrm{C}-\mathrm{B})$ & $\cdot$ & . & +.2 & . & . & . & . & $\mathrm{r}$ & $\mathrm{r}$ & 1.2 & + & . & III \\
\hline Potentilla arenaria & +.2 & . & . & . & . & . & + & +.2 & 2.2 & . & . & . & II \\
\hline Seseli annuит (C-B) & +.2 & . & . & $\mathrm{r}$ & . & + & + & . & $\cdot$ & . & . & . & II \\
\hline Thymus pulegioides (B) & +.2 & . & . & . & +.2 & . & . & . & +.2 & +.2 & . & . & II \\
\hline Ranunculus bulbosus (B) & + & + & . & + & $\cdot$ & . & . & . & . & $\cdot$ & . & . & II \\
\hline Allium oleraceum & . & . & . & . & . & . & . & $\mathrm{r}$ & . & . & . & +.2 & I \\
\hline Erigeron acris & . & $\mathrm{r}$ & . & $\mathrm{r}$ & . & . & . & . & . & . & . & $\cdot$ & I \\
\hline
\end{tabular}




\begin{tabular}{|c|c|c|c|c|c|c|c|c|c|c|c|c|c|}
\hline Successive No. & 1 & 2 & 3 & 4 & 5 & 6 & 7 & 8 & 9 & 10 & 11 & 12 & \\
\hline Field No. & 14 & 13 & 17 & 15 & 6 & 2 & 39 & 40 & 41 & 22 & 37 & 38 & \\
\hline \multirow{2}{*}{$\begin{array}{ll}\text { Date (2012) } & \text { day } \\
\text { month }\end{array}$} & 3 & 3 & 3 & 2 & 2 & 29 & 29 & 29 & 14 & 29 & 29 & 3 & $\stackrel{1}{7}$ \\
\hline & VI & VI & VI & VI & VI & VII & VII & VII & VII & VII & VII & VI & $\frac{1}{2}$ \\
\hline Slope exposure & NW & $\mathrm{N}$ & SE & SE & $\mathrm{SE}$ & $\mathrm{S}$ & SSE & SSE & SSW & $\mathrm{N}$ & $\mathrm{S}$ & $\mathrm{S}$ & E \\
\hline Inclination $\left[^{\circ}\right]$ & 5 & 7 & 5 & 3 & 7 & 5 & 10 & 3 & 10 & 3 & 3 & 5 & $\frac{\pi}{n}$ \\
\hline Density of shrub layer [\%] & 15 & 10 & 25 & 5 & 5 & 5 & 25 & 20 & 10 & 5 & + & 30 & తo \\
\hline Cover of herb layer $[\%]$ & 80 & 85 & 100 & 100 & 90 & 90 & 70 & 75 & 50 & 100 & 100 & 60 & \\
\hline Cover of moss layer [\%] & 80 & 60 & 5 & 10 & - & 70 & 50 & 70 & 80 & 20 & + & 40 & \\
\hline Area of relevé $\left[\mathrm{m}^{2}\right]$ & 15 & 15 & 25 & 10 & 20 & 8 & 15 & 25 & 20 & 20 & 25 & 20 & \\
\hline Number of taxa & 47 & 40 & 35 & 39 & 33 & 37 & 28 & 29 & 34 & 35 & 30 & 32 & \\
\hline Brachypodium pinnatum (B) & . & . & . & . & 4.5 & . & . & . & . & . & . & . & I \\
\hline \multicolumn{14}{|l|}{ V. Ch. Molinio-Arrhenatheretea } \\
\hline Arrhenatherum elatius & 2.1 & 2.2 & 1.1 & 1.1 & . & 1.1 & 1.1 & 1.1 & + & 1.1 & 2.1 & + & $\mathrm{V}$ \\
\hline Dactylis glomerata & + & + & +.2 & $+^{\circ}$ & $+^{\circ}$ & +.2 & . & . & . & +.2 & + & . & IV \\
\hline Festuca rubra & 1.1 & 1.2 & 2.1 & . & . & + & . & . & . & . & . & + & III \\
\hline Plantago lanceolata & + & + & . & . & . & . & + & . & $\mathrm{r}$ & + & . & $\mathrm{r}$ & III \\
\hline Veronica chamaedrys & . & . & . & . & $\mathrm{r}$ & $\mathrm{r}$ & . & . & . & +.2 & + & . & II \\
\hline Festuca pratensis & + & 1.2 & . & . & . & . & . & . & . & . & . & . & I \\
\hline Leontodon hispidus s.s. & + & + & . & . & . & . & . & . & . & . & . & . & I \\
\hline Prunella vulgaris & + & $\mathrm{r}$ & . & . & . & . & . & . & . & . & . & . & I \\
\hline Trifolium pratense & $\mathrm{r}$ & . & . & . & . & $+^{\circ}$ & . & . & . & . & . & . & I \\
\hline \multicolumn{14}{|l|}{ VI. Ch. Artemisietea vulgaris } \\
\hline Convolvulus arvensis & & & +.2 & + & & & $\mathrm{r}$ & & & + & + & $\mathrm{r}$ & III \\
\hline Linaria vulgaris & . & . & . & . & . & . & + & . & $+^{\circ}$ & . & + & + & II \\
\hline Daucus carota & $+^{\circ}$ & $+^{\circ}$ & . & . & . & . & . & . & . & . & + & + & II \\
\hline Picris hieracioides & . & . & + & + & . & . & . & . & . & . & $\mathrm{r}$ & + & II \\
\hline Artemisia vulgaris & . & + & . & . & . & . & . & . & . & . & . & $\mathrm{r}$ & I \\
\hline Glechoma hederacea & . & $\mathrm{r}$ & . & . & . & . & . & . & . & $\mathrm{r}$ & . & . & I \\
\hline \multicolumn{14}{|l|}{ VII. Ch. Rhamno-Prunetea } \\
\hline Prunus spinosa $\mathrm{b} / \mathrm{c}$ & 2.2 & 2.2 & 2.4 & + & + & 2.1 & 3.2 & 3.1 & . & 1.1 & + & 3.1 & $\mathrm{~V}$ \\
\hline Ligustrum vulgare b/c & + & + & + & 1.2 & + & 1.2 & . & +.2 & 1.2 & . & + & +.2 & $\mathrm{~V}$ \\
\hline Cornus sanguinea $\mathrm{b} / \mathrm{c}$ & 1.1 & 1.2 & +.2 & 1.2 & + & + & . & + & + & . & . & + & IV \\
\hline Rosa dumalis b/c & + & + & . & + & + & + & + & . & + & . & . & . & III \\
\hline Rhamnus catharticus $\mathrm{b} / \mathrm{c}$ & 1.1 & + & + & . & + & + & . & . & . & . & . & + & III \\
\hline Rosa canina $\mathrm{b} / \mathrm{c}$ & . & . & + & + & + & + & . & . & . & + & + & . & III \\
\hline Crataegus rhipidophylla b/c & . & $\mathrm{r}$ & . & . & . & . & . & . & . & + & . & . & I \\
\hline \multicolumn{14}{|l|}{ VIII. Ch. Querco-Fagetea } \\
\hline Acer pseudoplatanus b/c & . & . & $\mathrm{r}$ & + & . & . & . & . & . & . & . & . & I \\
\hline \multicolumn{14}{|l|}{ IX. Others } \\
\hline Juniperus communis b/c & 1.1 & 1.2 & . & + & 1.1 & $+^{\circ}$ & . & . & 2.2 & . & . & . & III \\
\hline Hieracium pilosella & +.2 & $\mathrm{r}$ & . & . & . & . & . & . & 1.2 & . & . & . & II \\
\hline Oxyrrhynchium hians d & . & . & . & 1.2 & . & . & . & . & . & +.2 & + & . & II \\
\hline Brachythecium rutabulum $\mathrm{d}$ & . & . & . & 2.2 & . & 1.2 & . & . & . & . & . & . & I \\
\hline Malus domestica b/c & + & . & . & . & . & . & . & + & . & . & . & . & I \\
\hline Prunus domestica b/c & . & . & . & . & . & . & + & . & + & . & . & . & I \\
\hline Calliergonella cuspidata $\mathrm{d}$ & 3.4 & . & . & . & . & . & . & . & . & . & . & . & I \\
\hline Thuidium philibertii $\mathrm{d}$ & 2.2 & . & . & . & . & . & . & . & . & . & . & . & $\mathrm{I}$ \\
\hline
\end{tabular}

Sporadic taxa: II. Anthericum ramosum 9(+); Trifolium alpestre 5(+); Viola collina 12(r); III. Clinopodium vulgare 10(1.2); Senecio jacobaea fo. 10(r); Silene nutans 4(+); IV. Anthyllis vulneraria 10(r); Artemisia campestris 10(+); Hieracium piloselloides 4(r); Onobrychis viciifolia (B) 1(+.2); Petrorhagia prolifera 12(+.2); Pimpinella saxifraga 1(+); V. Carex hirta 6(+); Carex spicata 6(+.2); Climacium dendroides d 1(+);Taraxacum officinale coll. 6(r); VI. Cichorium intybus 6(+); Euphorbia esula 2(r); Medicago lupulina 5(+.2); Melandrium album 4(r); Pastinaca sativa 4(+); VII. Pyrus pyraster b/c 10(+); Viburnum opulus b/c 1(+); VIII. Carex digitata 6(+); Carex montana 6(+.2); IX. Anthoxanthum odoratum 6(+); Calamagrostis epigejos 3(+); Cruciata glabra 10(1.1); Pinus sylvestris (V-P) 4(+); Plagiomnium affine d 6(+.2); Pteridium aquilinum 5(+); Quercus robur 9(+); Silene vulgaris 5(r); Stachys annua 12(r); Trifolium arvense (K-C) 11(r) 
Table 3. Floristic composition and differentiation of Geranio-Anemonetum sylvestris

\begin{tabular}{|c|c|c|c|c|c|c|c|c|c|c|c|c|}
\hline Successive No. & 1 & 2 & 3 & 4 & 5 & 6 & \multirow{11}{*}{ 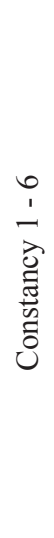 } & 7 & 8 & 9 & 10 & 11 \\
\hline Field No. & 24 & 28 & 23 & 33 & 34 & 32 & & 27 & 3 & 26 & 30 & 31 \\
\hline \multirow{2}{*}{ Date (2012) } & 19 & 21 & 19 & 28 & 28 & 28 & & 21 & 2 & 21 & 21 & 28 \\
\hline & VII & VII & VII & VII & VII & VII & & VII & VI & VII & VII & VII \\
\hline Slope exposure & - & SW & SSW & - & NW & NNE & & SW & SW & - & NW & - \\
\hline Inclination $\left[^{\circ}\right]$ & - & 15 & 3 & - & 30 & 30 & & 10 & 15 & - & 5 & - \\
\hline Density of shrub layer b [\%] & 20 & 10 & 30 & 25 & 25 & 40 & & 25 & + & 25 & 20 & 5 \\
\hline Cover of herb layer c [\%] & 90 & 70 & 70 & 65 & 50 & 50 & & 75 & 90 & 85 & 85 & 90 \\
\hline Cover of moss layer d [\%] & 90 & 90 & 75 & 75 & 35 & 90 & & 90 & 15 & 80 & 50 & 40 \\
\hline Area of relevé [m²] & 15 & 12 & 9 & 18 & 10 & 10 & & 12 & 20 & 16 & 20 & 15 \\
\hline Number of taxa & 30 & 33 & 23 & 30 & 35 & 32 & & 31 & 47 & 37 & 38 & 28 \\
\hline
\end{tabular}

I. Ch., *D. Ass.

Anemone sylvestris

*Sanguisorba minor (F-B)

Thalictrum minus

II. D. Subass.

Achillea pannonica $(\mathrm{F}-\mathrm{B})$

Potentilla arenaria $(\mathrm{F}-\mathrm{B})$

Veronica spicata (F-B)

Knautia arvensis fo. (T-G)

Sedum acre (K-C)

Centaurea stoebe (F-B)

Hieracium pilosella $(\mathrm{O})$

Cruciata glabra $(\mathrm{O})$

Carex flacca $(\mathrm{O})$

Trifolium pratense (M-A)

III. Ch., *D. Geranion sanguinei

Fragaria viridis

Vincetoxicum hirundinaria

*Galium boreale (M-A)

Primula veris

Viola collina

Vicia tenuifolia

Peucedanum cervaria

Anthericum ramosum

IV. Ch, *D. Origanetalia, Trifolio-Geranietea sanguinei

Poa angustifolia var. setacea

Agrimonia eupatoria

$\begin{array}{ccccccc}3.1 & 2.1 & 2.1 & 2.1 & 1.1 & 1.1 & \mathrm{~V} \\ 1.2 & 2.2 & 2.3 & \cdot & \cdot & + & \mathrm{IV} \\ 2.1 & 1.1 & \cdot & + & \cdot & \cdot & \mathrm{III} \\ + & \cdot & \cdot & \cdot & \cdot & \cdot & \mathrm{I} \\ + & \cdot & \cdot & + & + & + & \mathrm{IV} \\ \cdot & \cdot & \cdot & + & \cdot & + & \mathrm{II} \\ & + & & & & & \mathrm{I}\end{array}$

Galium album fo.

Galium verum fo.

Hypericum perforatum

Clinopodium vulgare

Coronilla varia

Viola hirta

\section{Ch. Festuco-Brometea}

Homalothecium lutescens $\mathrm{d}$

Abietinella abietina $\mathrm{d}$

Euphorbia cyparissias

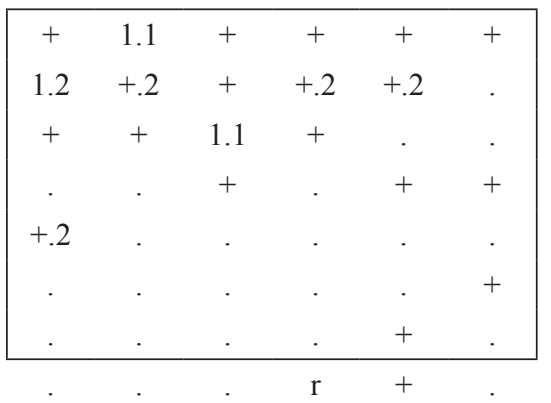

V
V
IV
III
I
I
I
II

$$
\text { I }
$$

\section{$\begin{array}{llllll}3.3 & 2.1 & 3.1 & 3.3 & 3.1 & 2.1\end{array}$}

V $\quad 3.3$ 


\begin{tabular}{|c|c|c|c|c|c|c|c|}
\hline \multirow{11}{*}{ 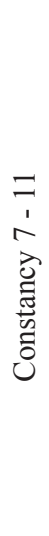 } & 12 & 13 & 14 & 15 & 16 & \multirow{11}{*}{ 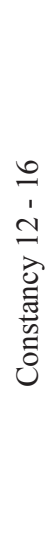 } & \multirow{11}{*}{ 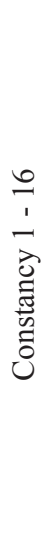 } \\
\hline & 20 & 29 & 21 & 19 & 25 & & \\
\hline & 3 & 21 & 14 & 3 & 19 & & \\
\hline & VI & VII & VII & VI & VII & & \\
\hline & - & SW & - & $\mathrm{N}$ & NNW & & \\
\hline & - & 5 & - & 35 & 6 & & \\
\hline & 10 & 20 & 10 & 20 & 5 & & \\
\hline & 75 & 50 & 60 & 50 & 80 & & \\
\hline & 20 & 90 & 80 & 70 & 60 & & \\
\hline & 8 & 12 & 8 & 15 & 15 & & \\
\hline & 34 & 33 & 31 & 41 & 27 & & \\
\hline
\end{tabular}

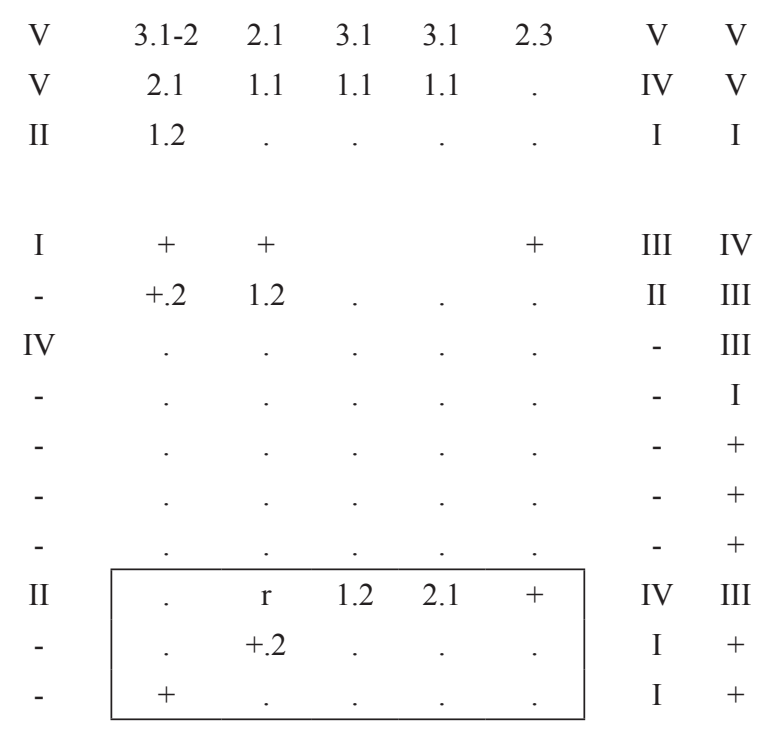

$\begin{array}{cccccccc}\text { V } & 3.1 & 2.1 & 1.1 & 2.1 & 3.1 & \text { V } & \text { V } \\ \text { III } & + & . & + & 1.1 & +.2 & \text { IV } & \text { IV } \\ \text { IV } & . & + & + & 1.1^{\circ} & 2.3 & \text { IV } & \text { IV } \\ \text { III } & . & + & . & . & 1.1 & \text { II } & \text { II } \\ \text { I } & . & . & . & . & . & - & \text { II } \\ \text { III } & . & . & . & . & . & - & \text { II } \\ \text { II } & . & + & . & . & . & \text { I } & \text { II } \\ \text { II } & . & . & . & . & . & - & \text { I }\end{array}$

$\begin{array}{cccccccc}\text { V } & 2.1 & + & 1.1 & + & 1.1 & \text { V } & \text { V } \\ \text { V } & + & 2.1 & + & + & + & \text { V } & \text { V } \\ \text { III } & \cdot & \cdot & + & + & + & \text { III } & \text { IV } \\ \text { III } & 2.1 & 1.1 & 1.1 & \cdot & \cdot & \text { III } & \text { IV } \\ \text { III } & + & \cdot & + & \cdot & \text { r } & \text { III } & \text { III } \\ \text { III } & + & + & 1.1 & 2.1 & + & \text { V } & \text { III } \\ \text { III } & \cdot & 1.1 & . & \cdot & . & \text { I } & \text { III } \\ \text { II } & . & 1.2 & . & . & + & \text { II } & \text { III }\end{array}$

$\begin{array}{cccccccc}\mathrm{V} & 2.2-3 & 3.3 & 1.2 & 2.2 & 3.3 & \mathrm{~V} & \mathrm{~V} \\ \mathrm{IV} & 1.2 & 3.3 & 4.4 & 4.4 & 3.3 & \mathrm{~V} & \mathrm{~V} \\ \mathrm{~V} & + & 1.1 & 1.1 & 1.1 & 1.1 & \mathrm{~V} & \mathrm{~V}\end{array}$




\begin{tabular}{|c|c|c|c|c|c|c|c|c|c|c|c|c|}
\hline Successive No. & 1 & 2 & 3 & 4 & 5 & 6 & \multirow{11}{*}{ 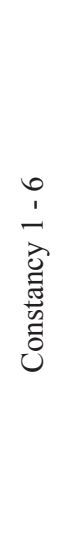 } & 7 & 8 & 9 & 10 & 11 \\
\hline Field No. & 24 & 28 & 23 & 33 & 34 & 32 & & 27 & 3 & 26 & 30 & 31 \\
\hline \multirow{2}{*}{$\begin{array}{ll}\text { Date (2012) } & \text { day } \\
\text { month }\end{array}$} & 19 & 21 & 19 & 28 & 28 & 28 & & 21 & 2 & 21 & 21 & 28 \\
\hline & VII & VII & VII & VII & VII & VII & & VII & VI & VII & VII & VII \\
\hline Slope exposure & - & SW & SSW & - & NW & NNE & & SW & SW & - & NW & - \\
\hline Inclination $\left[^{\circ}\right]$ & - & 15 & 3 & - & 30 & 30 & & 10 & 15 & - & 5 & - \\
\hline Density of shrub layer b [\%] & 20 & 10 & 30 & 25 & 25 & 40 & & 25 & + & 25 & 20 & 5 \\
\hline Cover of herb layer c [\%] & 90 & 70 & 70 & 65 & 50 & 50 & & 75 & 90 & 85 & 85 & 90 \\
\hline Cover of moss layer d [\%] & 90 & 90 & 75 & 75 & 35 & 90 & & 90 & 15 & 80 & 50 & 40 \\
\hline Area of relevé $\left[\mathrm{m}^{2}\right]$ & 15 & 12 & 9 & 18 & 10 & 10 & & 12 & 20 & 16 & 20 & 15 \\
\hline Number of taxa & 30 & 33 & 23 & 30 & 35 & 32 & & 31 & 47 & 37 & 38 & 28 \\
\hline Carex caryophyllea & 1.2 & + & 1.2 & 1.2 & . & 1.2 & $\mathrm{~V}$ & + & +.2 & +.2 & 1.1 & 1.2 \\
\hline Medicago falcata & 1.1 & 2.1 & 2.1 & 2.1 & + & $\mathrm{r}$ & $\mathrm{V}$ & 2.3 & 1.1 & + & $\cdot$ & $\cdot$ \\
\hline Thymus pulegioides (B) & +.2 & . & 1.2 & +.2 & +.2 & . & IV & . & . & +.2 & +.2 & . \\
\hline Polygala comosa (B) & $\cdot$ & . & . & $\cdot$ & + & . & I & . & + & $\mathrm{r}$ & $\mathrm{r}$ & . \\
\hline Seseli annuит (C-B) & + & . & 1.1 & . & . & . & II & . & . & . & . & + \\
\hline Melampyrum arvense (C-B) & . & . & $\mathrm{r}$ & . & . & + & II & . & . & . & . & $\mathrm{r}$ \\
\hline Achillea collina & . & + & . & . & . & . & I & + & $+^{\circ}$ & + & . & + \\
\hline Carlina vulgaris (B) & . & . & . & . & . & + & I & . & . & . & . & . \\
\hline Pimpinella saxifraga & + & + & . & . & $\mathrm{r}$ & & III & . & . & . & . & . \\
\hline Salvia pratensis & . & $\mathrm{r}$ & . & . & . & . & I & . & 1.2 & . & . & . \\
\hline Salvia verticillata $(\mathrm{C}-\mathrm{B})$ & . & . & $\cdot$ & . & . & . & - & . & & . & . & . \\
\hline Brachypodium pinnatum (B) & . & . & +.2 & . & . & . & I & . & 4.4 & . & . & . \\
\hline Centaurea scabiosa & . & . & +.2 & . & . & . & I & . & . & . & . & . \\
\hline Scabiosa ochroleuca & . & . & $\mathrm{r}$ & . & . & . & I & . & . & . & + & . \\
\hline Ranunculus bulbosus (B) & . & . & . & . & . & . & - & . & . & + & . & . \\
\hline
\end{tabular}

\section{Ch. Molinio-Arrhenatheretea}

Arrhenatherum elatius

Veronica chamaedrys

Plantago lanceolata

Dactylis glomerata

Carex hirta

Taraxacum officinale coll.

Festuca rubra

Leontodon hispidus s.s.

Lathyrus pratensis

\section{Ch. Artemisietea vulgaris}

Convolvulus arvensis

Medicago lupulina

\section{Ch. Rhamno-Prunetea}

Prunus spinosa $\mathrm{b} / \mathrm{c}$

Ligustrum vulgare b/c

Rhamnus catharticus b/c

Cornus sanguinea $\mathrm{b} / \mathrm{c}$

Rosa canina b/c

Rosa dumalis b/c

\section{Ch. Querco-Fagetea}

Euonymus verrucosus b/c

Carex montana

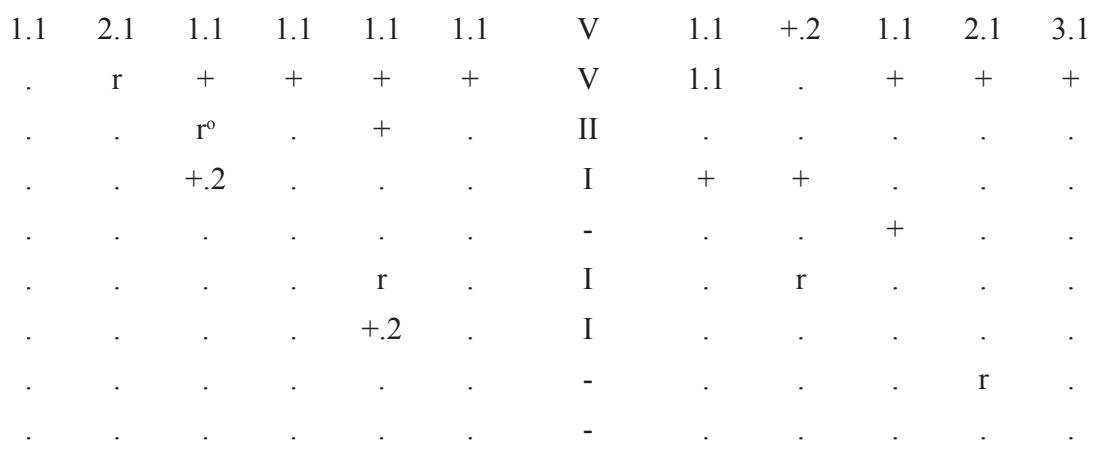

$\begin{array}{cccccccccccc}2.1 & 1.2 & 2.1 & + & 2.1 & 2.1 & \mathrm{~V} & + & 1.2 & + & + & . \\ 1.1 & 1.2 & + & 2.1 & . & 1.2 & \mathrm{~V} & 2.1 & 1.2 & + & . & + \\ . & + & + & 1.1 & . & 2.1 & \mathrm{IV} & + & +.2 & 2.2 & . & 1.1 \\ + & . & 2.1 & 2.1 & . & . & \mathrm{III} & . & . & + & 2.1 & + \\ . & + & . & + & + & 1.1 & \mathrm{IV} & . & . & . & + & . \\ . & . & . & . & . & . & - & + & + & . & . & .\end{array}$




\begin{tabular}{|c|c|c|c|c|c|c|c|}
\hline \multirow{11}{*}{ 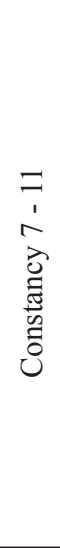 } & 12 & 13 & 14 & 15 & 16 & \multirow{11}{*}{ 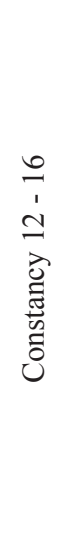 } & \multirow{11}{*}{$\begin{array}{l}0 \\
1 \\
3 \\
0 \\
0 \\
0 \\
0 \\
0\end{array}$} \\
\hline & 20 & 29 & 21 & 19 & 25 & & \\
\hline & 3 & 21 & 14 & 3 & 19 & & \\
\hline & VI & VII & VII & VI & VII & & \\
\hline & - & SW & - & $\mathrm{N}$ & NNW & & \\
\hline & - & 5 & - & 35 & 6 & & \\
\hline & 10 & 20 & 10 & 20 & 5 & & \\
\hline & 75 & 50 & 60 & 50 & 80 & & \\
\hline & 20 & 90 & 80 & 70 & 60 & & \\
\hline & 8 & 12 & 8 & 15 & 15 & & \\
\hline & 34 & 33 & 31 & 41 & 27 & & \\
\hline $\mathrm{V}$ & +.2 & 1.2 & 1.2 & . & 1.1 & IV & V \\
\hline III & 2.2 & 1.1 & . & . & . & II & IV \\
\hline II & +.2 & +.2 & +.2 & 1.2 & . & IV & IV \\
\hline III & + & . & $r$ & 1.1 & . & III & III \\
\hline I & 1.1 & + & . & . & . & II & II \\
\hline I & + & . & $r$ & . & . & II & II \\
\hline IV & . & . & . & . & . & - & II \\
\hline - & + & $r$ & . & + & . & II & II \\
\hline- & . & + & . & . & . & I & II \\
\hline I & . & . & . & + & . & I & I \\
\hline- & 1.2 & . & +.2 & +.2 & . & III & I \\
\hline I & . & . & $\cdot$ & . & . & - & I \\
\hline- & 1.2 & . & . & + & . & II & I \\
\hline I & $\cdot$ & . & . & . & . & - & I \\
\hline I & + & . & . & . & . & I & I \\
\hline $\mathrm{V}$ & 2.1 & 1.1 & 2.1 & 2.1 & 2.1 & $\mathrm{~V}$ & $\mathrm{~V}$ \\
\hline IV & +.2 & . & . & + & + & III & IV \\
\hline- & $+^{\circ}$ & . & . & $+^{\circ}$ & . & II & II \\
\hline II & $+^{0}$ & . & . & . & . & I & II \\
\hline I & . & + & . & . & +.2 & II & I \\
\hline I & . & $r$ & . & . & . & I & I \\
\hline- & +.2 & . & . & . & . & I & I \\
\hline I & . & + & . & . & . & I & I \\
\hline- & . & . & $+^{\circ}$ & $+^{\circ}$ & . & II & I \\
\hline II & . & . & . & . & + & I & II \\
\hline I & +.2 & . & $r$ & + & . & III & II \\
\hline IV & 2.1 & 2.1 & . & + & + & IV & $\mathrm{V}$ \\
\hline IV & . & 1.2 & + & + & . & III & IV \\
\hline IV & . & . & . & . & 1.1 & I & III \\
\hline III & + & . & 2.1 & 2.2 & . & III & III \\
\hline I & +.2 & + & . & . & . & II & III \\
\hline II & . & . & . & . & . & - & I \\
\hline II & . & . & . & . & . & - & I \\
\hline II & . & . & . & . & . & - & I \\
\hline
\end{tabular}




\begin{tabular}{|c|c|c|c|c|c|c|c|c|c|c|c|c|c|}
\hline \multicolumn{2}{|l|}{ Successive No. } & 1 & 2 & 3 & 4 & 5 & 6 & \multirow{11}{*}{ 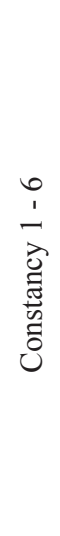 } & 7 & 8 & 9 & 10 & 11 \\
\hline \multicolumn{2}{|l|}{ Field No. } & 24 & 28 & 23 & 33 & 34 & 32 & & 27 & 3 & 26 & 30 & 31 \\
\hline \multirow{2}{*}{ Date (2012) } & day & 19 & 21 & 19 & 28 & 28 & 28 & & 21 & 2 & 21 & 21 & 28 \\
\hline & month & VII & VII & VII & VII & VII & VII & & VII & VI & VII & VII & VII \\
\hline \multicolumn{2}{|l|}{ Slope exposure } & - & SW & SSW & - & NW & NNE & & SW & SW & - & NW & - \\
\hline \multicolumn{2}{|c|}{ Inclination $\left[^{\circ}\right]$} & - & 15 & 3 & - & 30 & 30 & & 10 & 15 & - & 5 & - \\
\hline \multicolumn{2}{|c|}{ Density of shrub layer b [\%] } & 20 & 10 & 30 & 25 & 25 & 40 & & 25 & + & 25 & 20 & 5 \\
\hline \multicolumn{2}{|c|}{ Cover of herb layer c [\%] } & 90 & 70 & 70 & 65 & 50 & 50 & & 75 & 90 & 85 & 85 & 90 \\
\hline \multicolumn{2}{|c|}{ Cover of moss layer $d[\%]$} & 90 & 90 & 75 & 75 & 35 & 90 & & 90 & 15 & 80 & 50 & 40 \\
\hline \multicolumn{2}{|c|}{ Area of relevé $\left[\mathrm{m}^{2}\right]$} & 15 & 12 & 9 & 18 & 10 & 10 & & 12 & 20 & 16 & 20 & 15 \\
\hline \multicolumn{2}{|c|}{ Number of taxa } & 30 & 33 & 23 & 30 & 35 & 32 & & 31 & 47 & 37 & 38 & 28 \\
\hline
\end{tabular}

\section{Ch. Vaccinio-Piceetea}

Juniperus communis b/c

1.1

$2.1 \quad 2.1 \quad$ III

$+\quad 1.2^{\circ}$

Pleurozium schreberi d

Pinus sylvestris $\mathrm{b} / \mathrm{c}$

Pseudoscleropodium purum $\mathrm{d}$

Dicranum polysetum $\mathrm{d}$

\section{Others}

Thuidium philibertii d

Geranium pusillum

Silene vulgaris

Plagiomnium affine $\mathrm{d}$

Vicia hirsuta

Quercus robur b/c

Calamagrostis epigejos

Sporadic taxa: III. Campanula rapunculoides 8(1.2); Trifolium alpestre 8(+.2); IV. *Campanula persicifolia (Q-F) 8(+.2); Chamaecytisus ruthenicus 8(+.2); *Hieracium murorum (Q-F) 15(+.2); Origanum vulgare 8(1.2); *Rubus caesius var. arvalis (A) 14(+); Senecio jacobaea fo. 14(r); Vicia sepium 2(r); V. Allium oleraceum 15(r); Anthemis tinctoria 6(+); Anthyllis vulneraria 5(+); Aster amellus (C-B) 2(1.2); Orobanche caryophyllacea 8(+); Pimpinella nigra 7(r); Plantago media (B) 8(+); Verbascum lychnitis 10(+); VI. Avenula pubescens 12(+); Centaurea jacea 4(+); Climacium dendroides d $9(+)$; Festuca pratensis $12(+)$; Vicia cracca 12(+); VII. Cichorium intybus 13(+); Echium vulgare 14(r); Falcaria vulgaris 12(r $\left.\mathrm{r}^{\circ}\right)$; Glechoma hederacea 2(1.3); Linaria vulgaris 14(r); VIII. Crataegus monogyna b/c 4(+); Pyrus pyraster b/c 14(+); Rosa rubiginosa b/c 15(+); IX. Ajuga reptans 13(r); Carex digitata 8(+.2); Malus sylvestris b/c 9(+); XI. Fissidens sp. d 8(+.2); Oxyrrhynchium hians d 9(+); Quercus petraea b/c 15( $\left(^{\circ}\right)$ 


\begin{tabular}{|c|c|c|c|c|c|c|c|}
\hline \multirow{11}{*}{ 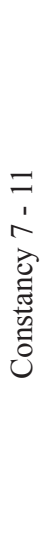 } & 12 & 13 & 14 & 15 & 16 & \multirow{11}{*}{ 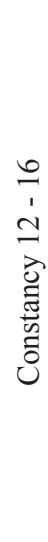 } & \multirow{11}{*}{$\begin{array}{l}0 \\
-1 \\
- \\
0 \\
0 \\
0 \\
0 \\
0\end{array}$} \\
\hline & 20 & 29 & 21 & 19 & 25 & & \\
\hline & 3 & 21 & 14 & 3 & 19 & & \\
\hline & VI & VII & VII & VI & VII & & \\
\hline & - & SW & - & $\mathrm{N}$ & NNW & & \\
\hline & - & 5 & - & 35 & 6 & & \\
\hline & 10 & 20 & 10 & 20 & 5 & & \\
\hline & 75 & 50 & 60 & 50 & 80 & & \\
\hline & 20 & 90 & 80 & 70 & 60 & & \\
\hline & 8 & 12 & 8 & 15 & 15 & & \\
\hline & 34 & 33 & 31 & 41 & 27 & & \\
\hline
\end{tabular}

\begin{tabular}{cccccccc}
$\mathrm{III}$ & +.2 & + & $\cdot$ & 1.1 & $\cdot$ & $\mathrm{III}$ & $\mathrm{III}$ \\
$\mathrm{I}$ & $\cdot$ & $\cdot$ & 1.3 & +.2 & $\cdot$ & $\mathrm{II}$ & $\mathrm{I}$ \\
- & $\cdot$ & $\cdot$ & $\cdot$ & + & $\cdot$ & $\mathrm{I}$ & $\mathrm{I}$ \\
$\mathrm{II}$ & $\cdot$ & $\cdot$ & $\cdot$ & $\cdot$ & $\cdot$ & - & $\mathrm{I}$ \\
- & $\cdot$ & $\cdot$ & $\cdot$ & 2.3 & $\cdot$ & $\mathrm{I}$ & $\mathrm{I}$ \\
& & & & & & & \\
$\mathrm{I}$ & +.2 & $\cdot$ & $\cdot$ & $\cdot$ & $\cdot$ & $\mathrm{I}$ & $\mathrm{II}$ \\
$\mathrm{II}$ & $\cdot$ & $\cdot$ & $\cdot$ & $\cdot$ & $\mathrm{r}$ & $\mathrm{I}$ & $\mathrm{II}$ \\
$\mathrm{II}$ & $\cdot$ & $\cdot$ & $\cdot$ & $\cdot$ & $\cdot$ & - & $\mathrm{II}$ \\
$\mathrm{III}$ & $\cdot$ & $\cdot$ & $\cdot$ & $\cdot$ & + & $\mathrm{I}$ & $\mathrm{II}$ \\
- & $\cdot$ & $\cdot$ & $\cdot$ & $\cdot$ & + & $\mathrm{I}$ & $\mathrm{II}$ \\
$\mathrm{I}$ & $\cdot$ & $\cdot$ & $\cdot$ & $\cdot$ & $\cdot$ & - & $\mathrm{I}$ \\
- & $\cdot$ & $\cdot$ & 2.1 & $\cdot$ & $\cdot$ & $\mathrm{I}$ & + \\
\hline
\end{tabular}


Table 4. Floristic composition of Lathyro sylvestris-Vincetoxicetum hirundinariae

\begin{tabular}{|c|c|c|c|c|c|c|}
\hline Successive No. & 1 & 2 & 3 & 4 & 5 & \multirow{11}{*}{$\begin{array}{l}n \\
3 \\
3 \\
0 \\
0 \\
0 \\
0 \\
0\end{array}$} \\
\hline Field No. & 51 & 5 & 18 & 50 & 49 & \\
\hline \multirow{2}{*}{ Date (2012) } & 2 & 3 & 18 & 18 & 18 & \\
\hline & VI & VI & VIII & VIII & VIII & \\
\hline Slope exposure & $\mathrm{S}$ & SSE & $\mathrm{SE}$ & SSW & $\mathrm{S}$ & \\
\hline Inclination $\left[^{\circ}\right]$ & 45 & 20 & 7 & 30 & 7 & \\
\hline Density of shrub layer [\%] & 5 & + & 20 & + & + & \\
\hline Cover of herb layer [\%] & 77 & 95 & 95 & 100 & 90 & \\
\hline Cover of moss layer[\%] & 80 & 20 & 30 & 90 & 95 & \\
\hline Area of relevé $\left[\mathrm{m}^{2}\right]$ & 10 & 18 & 15 & 25 & 20 & \\
\hline Number of taxa & 23 & 39 & 36 & 27 & 21 & \\
\hline \multicolumn{7}{|l|}{ I. Ch., *D. Geranion sanguinei } \\
\hline Vincetoxicum hirundinaria & 4.4 & 4.4 & 4.4 & 4.3 & 4.4 & $\mathrm{~V}$ \\
\hline Fragaria viridis & + & 2.1 & 2.1 & 2.1 & 1.1 & $\mathrm{~V}$ \\
\hline *Galium boreale (M-A) & + & 1.2 & 1.2 & 2.3 & + & $\mathrm{V}$ \\
\hline Peucedanum cervaria & $\mathrm{r}^{\mathrm{o}}$ & $\mathrm{r}$ & . & $+^{\circ}$ & . & III \\
\hline Vicia tenuifolia & $\mathrm{r}$ & . & 1.2 & . & . & II \\
\hline Anthericum ramosum & . & + & . & . & . & I \\
\hline Anemone sylvestris & . & $\mathrm{r}$ & . & . & . & I \\
\hline Geranium sanguineum & +.2 & . & . & . & . & I \\
\hline \multicolumn{7}{|l|}{ II. Ch. Origanetalia, Trifolio-Geranietea } \\
\hline Hypericum perforatum & + & + & + & + & + & $\mathrm{V}$ \\
\hline Agrimonia procera & + & + & . & + & $\mathrm{r}$ & IV \\
\hline Poa angustifolia var. setacea & . & + & 1.2 & + & 1.1 & IV \\
\hline Clinopodium vulgare & . & . & + & + & + & III \\
\hline Agrimonia eupatoria & $\mathrm{r}$ & . & 1.1 & + & . & III \\
\hline Galium album fo. & . & . & . & + & 1.1 & II \\
\hline Coronilla varia & . & 1.1 & . & + & . & II \\
\hline Astragalus glycyphyllos & $\mathrm{r}$ & $(+)$ & . & . & . & II \\
\hline \multicolumn{7}{|l|}{ III. Ch. Festuco-Brometea } \\
\hline Abietinella abietina $\mathrm{d}$ & 5.4 & 2.3 & 1.2 & 3.3 & 5.4 & $\mathrm{~V}$ \\
\hline Potentilla arenaria & $\mathrm{r}$ & +.2 & +.2 & +.2 & +.2 & $\mathrm{~V}$ \\
\hline Homalothecium lutescens d & +.2 & . & 3.3 & 3.3 & +.2 & IV \\
\hline Veronica spicata & + & +.2 & . & + & +.2 & IV \\
\hline Carex caryophyllea & + & 1.2 & +.2 & + & . & IV \\
\hline Medicago falcata & . & 1.2 & . & + & $\mathrm{r}$ & III \\
\hline Salvia pratensis & . & +.2 & +.2 & + & . & III \\
\hline Sanguisorba minor & + & . & + & + & . & III \\
\hline Centaurea scabiosa & . & . & 1.1 & +.2 & . & II \\
\hline Euphorbia cyparissias & + & + & . & . & . & II \\
\hline Melampyrum arvense (C-B) & . & . & + & . & $\mathrm{r}$ & II \\
\hline Thymus pulegioides (B) & . & +.2 & +.2 & . & . & II \\
\hline \multicolumn{7}{|l|}{ IV. Ch. Molinio-Arrhenatheretea } \\
\hline Arrhenatherum elatius & + & 1.2 & + & 2.1 & 1.1 & $\mathrm{~V}$ \\
\hline Veronica chamaedrys & + & $\mathrm{r}$ & + & . & + & IV \\
\hline Dactylis glomerata & . & + & $+^{\circ}$ & . & +.2 & III \\
\hline \multicolumn{7}{|l|}{ V. Ch. Rhamno-Prunetea } \\
\hline Rosa canina $\mathrm{b} / \mathrm{c}$ & + & + & + & + & . & IV \\
\hline Prunus spinosa $\mathrm{b} / \mathrm{c}$ & + & + & 2.2 & . & + & IV \\
\hline Rosa dumalis $\mathrm{b} / \mathrm{c}$ & . & + & + & . & $\mathrm{r}$ & III \\
\hline Rhamnus catharticus b/c & + & + & . & + & . & III \\
\hline Cornus sanguinea b/c & . & + & 1.2 & . & . & II \\
\hline Ligustrum vulgare $\mathrm{b} / \mathrm{c}$ & . & + & +.2 & . & . & II \\
\hline \multicolumn{7}{|l|}{ VI. Others } \\
\hline Convolvulus arvensis & . & 1.1 & . & . & + & II \\
\hline Elymus repens & . & + & $\mathrm{r}$ & . & . & II \\
\hline
\end{tabular}

Sporadic taxa: II. Chamaecytisus ruthenicus 2(+.2); Galium verum fo. 13(1.2); Knautia arvensis fo. 4(+); Origanum vulgare $2(+)$; Viola hirta $3(+)$; III. Achillea collina $4(+)$; Achillea pannonica $4(+)$; Brachypodium pinnatum (B) 2(1.3); Pimpinella saxifraga 3(r); Polygala comosa (B) 3(+); Ranunculus bulbosus (B)3(+); Salvia verticillata (C-B) 5(r); Scabiosa ochroleuca 2(1.2); Seseli annuum (C-B) 3(+.2); Thymus pannonicus $1(+.2)$; IV. Plantago lanceolata $3\left(+^{\circ}\right)$; V. Crataegus monogyna b/c 2(+); Pyrus pyraster b/c 2(+); VI. Glechoma hederacea $3(+)$; Calamagrostis epigejos $2(+)$; Hieracium pilosella $3(+.2)$; Juniperus communis b/c 2(+.2); Linum catharticum 3(+); Plagiomnium affine d 4(+); Quercus robur b/c 2( $\left.\mathrm{r}^{\circ}\right)$ 
Table 5. Floristic composition of Thalictro-Salvietum pratensis

\begin{tabular}{|c|c|c|c|c|c|c|c|c|}
\hline Successive No. & 1 & 2 & 3 & 4 & 5 & 6 & 7 & \\
\hline Field No. & 2 & 28 & 21 & 1 & 5 & 27 & 9 & \\
\hline \multirow{2}{*}{ Date (1993) } & 12 & 8 & 8 & 12 & 12 & 8 & 18 & 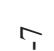 \\
\hline & 8 & 9 & 9 & 8 & 8 & 9 & 8 & $\frac{1}{2}$ \\
\hline Density of shrub layer [\%] & 20 & 10 & 5 & 10 & 10 & 10 & 20 & 运 \\
\hline Cover of herb layer [\%] & 100 & 100 & 100 & 100 & 100 & 100 & 100 & $\ddot{\forall}$ \\
\hline Cover of moss layer[\%] & 5 & - & 1 & 5 & - & - & - & ن \\
\hline Area of relevé $\left[\mathrm{m}^{2}\right]$ & 100 & 100 & 150 & 100 & 100 & 100 & 100 & \\
\hline Number of taxa & 63 & 48 & 48 & 55 & 42 & 52 & 45 & \\
\hline \multicolumn{9}{|l|}{ I. Ch. Cirsio-Brachypodion pinnati } \\
\hline Seseli annuum & 2 & 2 & 2 & 2 & 1 & 1 & 1 & $\mathrm{~V}$ \\
\hline Melampyrum arvense & 2 & . & 1 & 3 & 2 & 2 & . & IV \\
\hline Aster amellus & 1 & 3 & 1 & . & 1 & . & . & III \\
\hline Salvia verticillata & 1 & . & 2 & 2 & 1 & . & . & III \\
\hline Campanula sibirica & 1 & 1 & 1 & . & . & . & . & III \\
\hline \multicolumn{9}{|l|}{ II. Ch., *D. Brometalia erecti } \\
\hline Thymus pulegioides & 1 & 1 & 3 & 2 & . & 3 & 1 & $\mathrm{~V}$ \\
\hline *Medicago lupulina (A) & 1 & 2 & 2 & 1 & . & . & . & III \\
\hline Brachypodium pinnatum & 3 & 2 & . & . & . & . & . & II \\
\hline *Linum catharticum $(\mathrm{O})$ & + & + & . & . & . & . & . & II \\
\hline Carlina vulgaris & + & 1 & . & . & . & . & . & II \\
\hline \multicolumn{9}{|l|}{ III. Ch. Festuco-Brometea } \\
\hline Euphorbia cyparissias & 2 & 1 & 2 & 3 & 1 & 2 & 2 & $\mathrm{~V}$ \\
\hline Medicago falcata & 2 & + & 1 & 2 & 2 & 2 & 2 & $\mathrm{~V}$ \\
\hline Scabiosa ochroleuca & 1 & 1 & 2 & 2 & 2 & 2 & 1 & $\mathrm{~V}$ \\
\hline Veronica spicata & 2 & 1 & 2 & 2 & 3 & 3 & 3 & $\mathrm{~V}$ \\
\hline Sanguisorba minor & 2 & 2 & 2 & 2 & 1 & 3 & + & $\mathrm{V}$ \\
\hline Verbascum lychnitis & 1 & 1 & 1 & 1 & 1 & 2 & + & $\mathrm{V}$ \\
\hline Anthemis tinctoria & 2 & . & 1 & + & 1 & 1 & 1 & $\mathrm{~V}$ \\
\hline Anthyllis vulneraria & 1 & 2 & 1 & + & . & 1 & . & IV \\
\hline Centaurea scabiosa & . & . & 1 & 1 & 1 & 2 & . & III \\
\hline Bromus inermis fo. & 3 & . & . & 1 & 2 & . & 2 & III \\
\hline Centaurea stoebe & 1 & 2 & . & . & 1 & 2 & . & III \\
\hline Senecio jacobaea & 1 & . & 1 & 1 & . & . & + & III \\
\hline Abietinella abietina $\mathrm{d}$ & 1 & . & 1 & 1 & . & . & . & III \\
\hline Pimpinella saxifraga & 1 & . & . & 2 & 1 & . & . & III \\
\hline Carex caryophyllea & 1 & 1 & . & . & . & 1 & . & III \\
\hline Poa compressa & . & . & + & . & + & 1 & . & III \\
\hline Acinos arvensis & . & 1 & . & + & . & 1 & . & III \\
\hline Potentilla arenaria & . & 2 & + & . & . & . & . & II \\
\hline Achillea pannonica & 1 & . & . & . & . & 1 & . & II \\
\hline Salvia pratensis & 2 & . & . & . & 2 & . & . & II \\
\hline Stachys recta & . & 1 & . & + & . & . & . & II \\
\hline Arenaria serpyllifolia & . & . & . & . & . & 2 & . & I \\
\hline Artemisia campestris & . & . & 1 & . & . & . & . & I \\
\hline Petrorhagia prolifera & . & . & . & 1 & . & . & . & I \\
\hline \multicolumn{9}{|l|}{ IV. Ch. Trifolio-Geranietea sanguinei } \\
\hline Vincetoxicum hirundinaria $(\mathrm{G})$ & 1 & 1 & + & + & 1 & 2 & 1 & $\mathrm{~V}$ \\
\hline Hypericum perforatum & + & 1 & + & 1 & 1 & 1 & + & $\mathrm{V}$ \\
\hline Agrimonia procera & 2 & 1 & 2 & 2 & 2 & 1 & 2 & $\mathrm{~V}$ \\
\hline Fragaria viridis $(\mathrm{G})$ & 3 & 2 & 3 & 3 & 3 & 3 & . & $\mathrm{V}$ \\
\hline Anemone sylvestris $(\mathrm{G})$ & 2 & 2 & 2 & . & 1 & 2 & 1 & $\mathrm{~V}$ \\
\hline Poa angustifolia var. setacea & . & 1 & 3 & . & 2 & 3 & 2 & IV \\
\hline Gallium album fo. & 1 & . & . & 2 & 2 & + & 1 & IV \\
\hline Galium verum fo. & 2 & . & 2 & 1 & . & 1 & 2 & IV \\
\hline Coronilla varia & + & . & 3 & 2 & . & 2 & 2 & IV \\
\hline Anthericum ramosum $(\mathrm{G})$ & 1 & 3 & 1 & + & . & + & . & IV \\
\hline Peucedanum cervaria $(\mathrm{G})$ & 2 & 3 & . & . & 2 & 2 & . & III \\
\hline
\end{tabular}




\begin{tabular}{|c|c|c|c|c|c|c|c|c|}
\hline Successive No. & 1 & 2 & 3 & 4 & 5 & 6 & 7 & \multirow{9}{*}{ 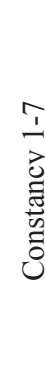 } \\
\hline Field No. & 2 & 28 & 21 & 1 & 5 & 27 & 9 & \\
\hline \multirow{2}{*}{ Date (1993) } & 12 & 8 & 8 & 12 & 12 & 8 & 18 & \\
\hline & 8 & 9 & 9 & 8 & 8 & 9 & 8 & \\
\hline Density of shrub layer [\%] & 20 & 10 & 5 & 10 & 10 & 10 & 20 & \\
\hline Cover of herb layer [\%] & 100 & 100 & 100 & 100 & 100 & 100 & 100 & \\
\hline Cover of moss layer[\%] & 5 & - & 1 & 5 & - & - & - & \\
\hline Area of relevé [m²] & 100 & 100 & 150 & 100 & 100 & 100 & 100 & \\
\hline Number of taxa & 63 & 48 & 48 & 55 & 42 & 52 & 45 & \\
\hline Clinopodium vulgare & + & . & . & + & 2 & . & 1 & III \\
\hline Thalictrum minus s.s. $(\mathrm{G})$ & 1 & . & . & 2 & 1 & . & . & III \\
\hline Vicia tenuifolia $(\mathrm{G})$ & 1 & . & . & . & . & 1 & + & III \\
\hline Viola hirta & + & + & . & . & . & . & . & II \\
\hline \multicolumn{9}{|l|}{ V. Ch. Molinio-Arrhenatheretea } \\
\hline Galium boreale & 3 & 3 & 3 & . & 3 & 3 & 3 & $\mathrm{~V}$ \\
\hline Arrhenatherum elatius & 2 & . & 2 & 1 & 3 & 1 & 2 & $\mathrm{~V}$ \\
\hline Knautia arvensis & . & 1 & 1 & 1 & . & 1 & + & IV \\
\hline Plantago lanceolata & 1 & + & 1 & 1 & . & 1 & . & IV \\
\hline Dactylis glomerata & 1 & . & 2 & 1 & . & + & 1 & IV \\
\hline Achillea millefolium & 1 & . & 2 & 2 & 1 & . & . & III \\
\hline Potentilla reptans & 1 & . & . & 1 & . & . & . & II \\
\hline Rumex acetosa & . & . & . & + & . & + & . & II \\
\hline Poa pratensis & 1 & . & . & 2 & . & . & . & II \\
\hline Lotus corniculatus & . & . & 1 & . & . & . & . & I \\
\hline Carex hirta & . & . & . & 1 & . & . & . & I \\
\hline Crepis biennis & . & . & . & . & 1 & . & . & I \\
\hline Inula salicina & . & . & . & . & . & . & 1 & I \\
\hline \multicolumn{9}{|l|}{ VI. Ch. Koelerio-Corynephoretea } \\
\hline Trifolium campestre & . & . & + & 1 & + & . & + & III \\
\hline Trifolium arvense & . & . & . & 2 & . & . & . & I \\
\hline Cerastium arvense & 1 & . & . & . & . & . & . & I \\
\hline
\end{tabular}

VII. Ch. Artemisietea vulgaris

Convolvulus arvensis

Carduus acanthoides

Echium vulgare

Picris hieracioides

Linaria vulgaris

Elymus repens

VIII. Ch. Rhamno-Prunetea

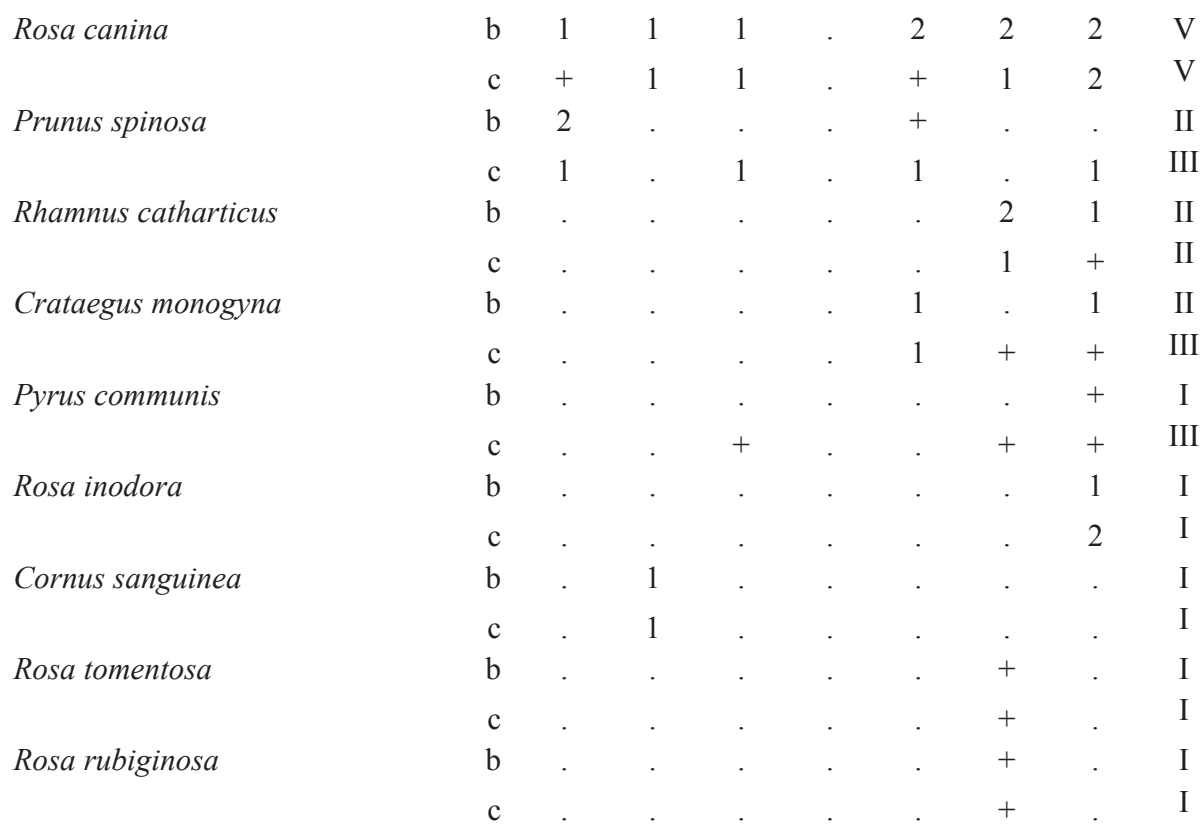




\begin{tabular}{|c|c|c|c|c|c|c|c|c|c|}
\hline Successive No. & & 1 & 2 & 3 & 4 & 5 & 6 & 7 & \\
\hline Field No. & & 2 & 28 & 21 & 1 & 5 & 27 & 9 & \\
\hline \multirow{2}{*}{ Date (1993) } & day & 12 & 8 & 8 & 12 & 12 & 8 & 18 & $\uparrow$ \\
\hline & month & 8 & 9 & 9 & 8 & 8 & 9 & 8 & $\vec{\lambda}$ \\
\hline Density of shrub layer [\%] & & 20 & 10 & 5 & 10 & 10 & 10 & 20 & $\stackrel{\Xi}{\pi}$ \\
\hline Cover of herb layer [\%] & & 100 & 100 & 100 & 100 & 100 & 100 & 100 & \\
\hline Cover of moss layer $[\%]$ & & 5 & - & 1 & 5 & - & - & - & ن \\
\hline Area of relevé [m²] & & 100 & 100 & 150 & 100 & 100 & 100 & 100 & \\
\hline Number of taxa & & 63 & 48 & 48 & 55 & 42 & 52 & 45 & \\
\hline
\end{tabular}

\section{Ch. Querco-Fagetea}

Eunoymus verrucosus

b 1

\section{Ch. Vaccinio-Piceetea}

Juniperus communis

Pinus sylvestris

Melampyrum pratense

\section{Others}

Quercus petraea

Silene vulgaris

Pteridium aquilinum

Hieracium pilosella

Primula veris

Geranium columbinum

Calamagrostis epigejos

Cerinthe minor

Briza media

Agrostis capillaris

c +

I

I

$\begin{array}{ccccccccc}\mathrm{b} & 1 & 2 & 1 & . & . & 1 & 2 & \mathrm{IV} \\ \mathrm{c} & + & + & 1 & . & . & + & 1 & \mathrm{IV} \\ \mathrm{b} & + & 2 & . & . & . & . & . & \mathrm{II} \\ \mathrm{c} & + & 2 & . & . & . & . & . & \text { II } \\ & . & . & . & . & . & . & 2 & \mathrm{I}\end{array}$

Sporadic taxa: II: Gentianella ciliata 2(+); Ranunculus bulbosus 1(+); III: Viola rupestris 2(+); Orobanche caryophyllacea 1(+); Erigeron acris 1(+); IV: Geranium sanguineum $2(+)$; Veronica teucrium $7(+)$; Fragaria vesca $4(+)$; V: Taraxacum officinale coll. 5(+); Rhinanthus serotinus $7(+)$; Veronica chamaedrys 4(+); Leontodon hispidus s.1. 7(+); Trifolium pratense 2(+); VI: Sedum acre 6(+); VII: Ligustrum vulgare b 1(+); VIII: Digitalis grandiflora 5(+); X: Sedum maximum 4(+); Myosotis arvensis 4(+); Convallaria majalis 7(+); Polygala vulgaris 1(+); Lychnis viscaria 2(+); Malus domestica 5(+) 
Table 6. Synoptic table of xerothermic communities studied in the years 1993 (col. 6) and 2012 (col. 1-5)

\begin{tabular}{lcccccc}
\hline Successive No. & 1 & 2 & 3 & 4 & 5 & 6 \\
Abbreviated syntaxon name & $\mathrm{P}$ & $\mathrm{C}-\mathrm{V}$ & $\mathrm{G}-\mathrm{A}$ & $\mathrm{L}-\mathrm{V}$ & $\mathrm{G}$ coll. & TS \\
Number of relevés & 10 & 12 & 16 & 5 & 43 & 7 \\
\hline
\end{tabular}

\section{Ch., *D. Ass.}

Peucedanum cervaria

*Aster amellus (C-B)

Vicia tenuifolia

*Falcaria vulgaris (A)

Campanula bononiensis

*Sanguisorba minor (F-B)

Anemone sylvestris

Thalictrum minus

II. Ch. Cirsio-Brachypodion pinnati

Seseli annuum

Melampyrum arvense

Salvia verticillata

Campanula sibirica

III. Ch., *D. Geranion sanguinei

Fragaria viridis

*Galium boreale (M-A)

Vincetoxicum hirundinaria

Primula veris

Anthericum ramosum

Viola collina

Geranium sanguineum

Campanula rapunculoides

Trifolium alpestre

Trifolium rubens

Veronica teucrium

IV. Ch., *D. Brometalia erecti

Polygala comosa

Carex flacca

Thymus pulegioides

*Medicago lupulina (A)

*Linum catharticum (O)

Brachypodium pinnatum

Gentianella cilliata

Carlina vulgaris

Ranunculus bulbosus

Plantago media

Onobrychis viciifolia

Alchemilla glaucescens

V. Ch. Festuco-Brometea

Homalothecium lutescens $\mathrm{d}$

Achillea collina

Allium oleraceum

Euphorbia cyparissias

Medicago falcata

Carex caryophyllea

Abietinella abietina d

Potentilla arenaria

Centaurea scabiosa

Salvia pratensis

Achillea pannonica

Pimpinella saxifraga

Veronica spicata

Scabiosa ochroleuca

Anthyllis vulneraria

\begin{tabular}{|c|c|c|c|c|}
\hline$V^{2-4}$ & $\mathrm{II}^{+-1}$ & $\mathrm{II}^{+-2}$ & $\mathrm{IIII}^{\mathrm{r}-+}$ & $\mathrm{IIII}^{\mathrm{r}-4}$ \\
\hline $\mathrm{II}^{+-1}$ & - & $\mathrm{I}^{1}$ & - & $\mathrm{I}^{+-1}$ \\
\hline $\mathrm{II}^{+}$ & $\mathrm{V}^{2-5}$ & $\mathrm{II}^{\mathrm{r}-+}$ & $\mathrm{II}^{\mathrm{r}-1}$ & $\mathrm{IIII}^{\mathrm{r}-5}$ \\
\hline$+^{r}$ & $\mathrm{II}^{+-1}$ & $+^{r}$ & - & $\mathrm{I}^{\mathrm{r}-2}$ \\
\hline- & $+^{+}$ & - & - & $\mathrm{r}^{+}$ \\
\hline $\mathrm{V}^{\mathrm{r}-\mathrm{t}}$ & $\mathrm{V}^{+-1}$ & $\mathrm{~V}^{\mathrm{r}-2}$ & $\mathrm{III}^{+}$ & $\mathrm{V}^{\mathrm{r}-2}$ \\
\hline $\mathrm{II}^{\mathrm{r}-1}$ & - & $\mathrm{V}^{2-3}$ & $\mathrm{I}^{\mathrm{r}}$ & $\mathrm{IIII}^{\mathrm{r}-3}$ \\
\hline $\mathrm{II}^{\mathrm{r}-\mathrm{t}}$ & $\mathrm{II}^{+}$ & $\mathrm{I}^{+-1}$ & - & $\mathrm{II}^{\mathrm{r}-1}$ \\
\hline
\end{tabular}

\begin{tabular}{lcccc|c|}
\hline $\mathrm{IV}^{\mathrm{r}-+}$ & $\mathrm{II}^{\mathrm{r}-+}$ & $\mathrm{II}^{+-1}$ & $\mathrm{I}^{+}$ & $\mathrm{III}^{\mathrm{r}-1}$ & $\mathrm{~V}^{1-2}$ \\
$\mathrm{II}^{\mathrm{r}-+}$ & $\mathrm{III}^{\mathrm{r}-1}$ & $\mathrm{II}^{\mathrm{r}-+}$ & $\mathrm{II}^{\mathrm{r}-+}$ & $\mathrm{III}^{\mathrm{r}-1}$ & $\mathrm{IV}^{1-3}$ \\
$\mathrm{II}^{\mathrm{r}-+}$ & $\mathrm{III}^{\mathrm{r}-1}$ & $\mathrm{I}^{+-1}$ & $+\mathrm{r}$ & $\mathrm{II}^{\mathrm{r}-1}$ & $\mathrm{III}^{1-2}$ \\
$\left(\mathrm{I}^{+}\right)$ & - & - & - & $\left(\mathrm{r}^{+}\right)$ & $\mathrm{III}^{1}$ \\
\cline { 3 - 5 }
\end{tabular}

$\begin{array}{cccccc}\mathrm{V}^{\mathrm{r}-2} & \mathrm{~V}^{+-3} & \mathrm{~V}^{1-3} & \mathrm{~V}^{+-2} & \mathrm{~V}^{\mathrm{r}-3} & \mathrm{~V}^{2-3} \\ \mathrm{~V}^{+-3} & \mathrm{III}^{\mathrm{r}-1} & \mathrm{IV}^{+-2} & \mathrm{~V}^{+-2} & \mathrm{IV}^{\mathrm{r}-3} & \mathrm{~V}^{3} \\ \mathrm{III}^{+-2} & \mathrm{II}^{+-1} & \mathrm{IV}^{+-2} & \mathrm{~V}^{4} & \mathrm{III}^{+-4} & \mathrm{~V}^{+-2} \\ \mathrm{I}^{+-2} & \mathrm{II}^{\mathrm{r}-2} & \mathrm{II}^{\mathrm{r}-2} & - & \mathrm{II}^{\mathrm{r}-2} & \mathrm{II}^{+-1} \\ \mathrm{II}^{+-2} & +^{+} & \mathrm{I}^{+-1} & \mathrm{I}^{+} & \mathrm{I}^{+-1} & \mathrm{IV}^{+-3} \\ \mathrm{II}^{\mathrm{r}-2} & +^{\mathrm{r}} & \mathrm{II}^{+-1} & - & \mathrm{II}^{\mathrm{r}-2} & - \\ \mathrm{I}^{2} & - & - & \mathrm{I}^{+} & +^{+-2} & \mathrm{I}^{+} \\ - & \mathrm{I}^{+} & \mathrm{I}^{1} & - & \mathrm{I}^{+-1} & - \\ \mathrm{I}^{+} & +^{+} & +^{+} & - & +^{+} & - \\ \mathrm{I}^{+} & - & - & - & \mathrm{r}^{+} & - \\ - & - & - & - & - & \mathrm{I}^{+}\end{array}$

\begin{tabular}{|ccccc|c|}
\hline $\mathrm{II}^{\mathrm{r}-+}$ & - & $\mathrm{III}^{\mathrm{r}-1}$ & $\mathrm{I}^{+}$ & $\mathrm{II}^{\mathrm{r}-1}$ & - \\
$\mathrm{II}^{+-1}$ & - & $+^{+}$ & - & $\mathrm{I}^{+-1}$ & - \\
\cline { 1 - 4 } $\mathrm{II}^{+}$ & $\mathrm{II}^{+}$ & $\mathrm{IV}^{+-1}$ & $\mathrm{II}^{+}$ & $\mathrm{III}^{+-1}$ & $\mathrm{~V}^{1-3}$ \\
$\mathrm{I}^{\mathrm{r}-}$ & $+^{+}$ & $\mathrm{II}^{\mathrm{r}-1}$ & - & $\mathrm{I}^{\mathrm{r}-1}$ & $\mathrm{III}^{1-2}$ \\
$\mathrm{II}^{\mathrm{r}-+}$ & - & - & $\mathrm{I}^{+}$ & $\mathrm{I}^{\mathrm{r}-+}$ & $\mathrm{III}^{+}$ \\
$\mathrm{I}^{3-4}$ & $\mathrm{I}^{4}$ & $\mathrm{I}^{+-4}$ & $\mathrm{I}^{1}$ & $\mathrm{I}^{+-4}$ & $\mathrm{II}^{2-3}$ \\
- & - & - & - & - & $\mathrm{I}^{+}$ \\
$\mathrm{II}^{\mathrm{r}-+}$ & $\mathrm{III}^{+}$ & $\mathrm{II}^{\mathrm{r}-+}$ & - & $\mathrm{II}^{\mathrm{r}-+}$ & $\mathrm{II}^{+-1}$ \\
$\mathrm{I}^{+}$ & $\mathrm{II}^{+}$ & $\mathrm{I}^{+}$ & $\mathrm{I}^{+}$ & $\mathrm{I}^{+}$ & $\mathrm{I}^{+}$ \\
$\mathrm{I}^{+}$ & - & $+^{+}$ & - & $\mathrm{r}^{+}$ & - \\
- & $+^{+}$ & - & - & $\mathrm{r}^{+}$ & - \\
$\mathrm{I}^{+}$ & - & - & - & $\mathrm{r}^{+}$ & -
\end{tabular}

\begin{tabular}{|c|c|c|c|c|c|}
\hline $\mathrm{V}^{+-4}$ & $\mathrm{~V}^{+-4}$ & $\mathrm{~V}^{+-4}$ & $\mathrm{IV}^{+-3}$ & $\mathrm{~V}^{+-4}$ & - \\
\hline $\mathrm{III}^{+-1}$ & $\mathrm{IV}^{\mathrm{r}-1}$ & $\mathrm{II}^{+}$ & - & $\mathrm{III}^{\mathrm{r}-1}$ & - \\
\hline $\mathrm{I}^{+}$ & $\mathrm{I}^{\mathrm{r}-\mathrm{+}}$ & $+^{r}$ & - & $\mathrm{I}^{\mathrm{r}-+}$ & - \\
\hline $\mathrm{V}^{+-1}$ & $\mathrm{IV}^{\mathrm{r}-+}$ & $\mathrm{V}^{+-1}$ & $\overline{\mathrm{II}^{+}}$ & $\mathrm{V}^{\mathrm{r}-1}$ & $\mathrm{~V}^{1-3}$ \\
\hline $\mathrm{IV}^{+-1}$ & $\mathrm{IIII}^{\mathrm{r}-1}$ & $\mathrm{IV}^{\mathrm{r}-2}$ & $\mathrm{III}^{\mathrm{r}-1}$ & $\mathrm{IV}^{\mathrm{r}-2}$ & $\mathrm{~V}^{+-2}$ \\
\hline $\mathrm{IV}^{+-1}$ & $\mathrm{III}^{+-1}$ & $\mathrm{~V}^{+-1}$ & $\mathrm{IV}^{+-1}$ & $\mathrm{IV}^{+-1}$ & $\mathrm{III}^{1-2}$ \\
\hline $\mathrm{V}^{+-4}$ & $\mathrm{III}^{2}$ & $\mathrm{~V}^{+-5}$ & $V^{1-5}$ & $\mathrm{~V}^{+-5}$ & $\mathrm{III}^{1}$ \\
\hline $\mathrm{IV}^{+-1}$ & $\mathrm{II}^{+-2}$ & $\mathrm{III}^{+-1}$ & $\mathrm{~V}^{\mathrm{r}-+}$ & $\mathrm{III}^{\mathrm{r}-2}$ & $\mathrm{II}^{+-2}$ \\
\hline $\mathrm{II}^{+-1}$ & $\mathrm{IV}^{+-2}$ & $\mathrm{I}^{+-1}$ & $\mathrm{II}^{+-1}$ & $\mathrm{III}^{+-2}$ & III $^{1-2}$ \\
\hline $\mathrm{IV}^{+-2}$ & $\mathrm{III}^{+-1}$ & $\mathrm{I}^{\mathrm{r}-1}$ & $\mathrm{III}^{+}$ & $\mathrm{III}^{\mathrm{r}-2}$ & $\mathrm{II}^{2}$ \\
\hline $\mathrm{II}^{+}$ & - & $\mathrm{IV}^{+-1}$ & $\mathrm{I}^{+}$ & $\mathrm{II}^{+-1}$ & II $^{1}$ \\
\hline$+^{r}$ & $\mathrm{III}^{+-1}$ & $\mathrm{II}^{\mathrm{r}-+}$ & $\mathrm{I}^{\mathrm{r}}$ & $\mathrm{I}^{\mathrm{r}-1}$ & $\mathrm{III}^{+-1}$ \\
\hline $\mathrm{IV}^{+-1}$ & - & $\mathrm{III}^{\mathrm{r}-+}$ & $\mathrm{IV}^{+}$ & $\mathrm{III}^{\mathrm{r}-1}$ & $\mathrm{~V}^{1-3}$ \\
\hline $\mathrm{IV}^{\mathrm{r}-+}$ & $\mathrm{III}^{\mathrm{r}-+}$ & $\mathrm{I}^{\mathrm{r}-+}$ & $\mathrm{I}^{1}$ & $\mathrm{II}^{\mathrm{r}-1}$ & $\mathrm{~V}^{1-2}$ \\
\hline $\mathrm{II}^{+}$ & $+^{r}$ & $+^{+}$ & - & $\mathrm{I}^{\mathrm{r}-+}$ & $\mathrm{IV}^{+-2}$ \\
\hline
\end{tabular}




\begin{tabular}{|c|c|c|c|c|c|c|}
\hline Successive No. & 1 & 2 & 3 & 4 & 5 & 6 \\
\hline Abbreviated syntaxon name & $\mathrm{P}$ & $\mathrm{C}-\mathrm{V}$ & G-A & L-V & G coll. & TS \\
\hline Number of relevés & 10 & 12 & 16 & 5 & 43 & 7 \\
\hline Verbascum lychnitis & - & - & $+^{+}$ & - & $\mathrm{r}^{+}$ & $\mathrm{V}^{+-2}$ \\
\hline Anthemis tinctoria & - & - & $+^{+}$ & - & $\mathrm{r}^{+}$ & $\mathrm{V}^{+-2}$ \\
\hline Centaurea stoebe & $\mathrm{I}^{\mathrm{r}-+}$ & - & $+^{+}$ & - & $++^{r-+}$ & IIII $^{1-2}$ \\
\hline Bromus inermis & - & - & - & - & - & $\mathrm{III}^{1-3}$ \\
\hline Poa compressa & - & - & - & - & - & $\mathrm{III}^{+-1}$ \\
\hline Acinos arvensis & - & - & - & - & - & $\mathrm{III}^{+-1}$ \\
\hline Stachys recta & $\mathrm{I}^{+}$ & - & - & - & $\mathrm{r}^{+}$ & $\mathrm{II}^{+-1}$ \\
\hline Arenaria serpyllifolia & - & - & - & - & - & $\mathrm{I}^{2}$ \\
\hline Viola rupestris & - & - & - & - & - & $\mathrm{I}^{+}$ \\
\hline Petrorhagia prolifera & $\mathrm{I}^{\mathrm{r}}$ & $+^{+}$ & - & - & $+^{r-+}$ & $\mathrm{I}^{1}$ \\
\hline Orobanche caryophyllacea & $+^{r}$ & - & $+^{+}$ & - & $\mathrm{r}^{\mathrm{r}-+}$ & $\mathrm{I}^{+}$ \\
\hline Erigeron acris & - & $\mathrm{I}^{\mathrm{r}}$ & - & - & $\mathrm{r}^{\mathrm{r}}$ & $\mathrm{I}^{+}$ \\
\hline Artemisia campestris & - & $+^{+}$ & - & - & $\mathrm{r}^{+}$ & $\mathrm{I}^{1}$ \\
\hline Phleum phleoides & $\mathrm{I}^{+}$ & - & - & - & $\mathrm{r}^{+}$ & - \\
\hline Thymus pannonicus ssp. marschallianus & $\mathrm{I}^{1}$ & - & - & - & $++^{1}$ & - \\
\hline Thymus pannonicus & - & - & - & $\mathrm{I}^{+}$ & $+^{+}$ & - \\
\hline Hieracium piloselloides & - & $+^{\mathrm{r}}$ & - & - & $\mathrm{r}^{\mathrm{r}}$ & - \\
\hline Arabis hirsuta & $+\mathrm{r}$ & - & - & - & $\mathrm{r}^{\mathrm{r}}$ & - \\
\hline \multicolumn{7}{|c|}{$\begin{array}{l}\text { VI. Ch., *D. Origanetalia, Trifolio-Geranietea } \\
\text { sanguinei }\end{array}$} \\
\hline Agrimonia eupatoria & $\mathrm{IV}^{+-2}$ & $\mathrm{IV}^{+-1}$ & $\mathrm{~V}^{+-2}$ & $\mathrm{III}^{\mathrm{r}-1}$ & $\mathrm{IV}^{\mathrm{r}-2}$ & - \\
\hline *Rubus caesius var. arvalis (A) & $\mathrm{II}^{+-1}$ & $\mathrm{~V}^{+-4}$ & $+^{+}$ & - & $\mathrm{II}^{+-4}$ & - \\
\hline Astragalus glycyphyllos & $\mathrm{III}^{+}$ & $\mathrm{II}^{+}$ & - & $\mathrm{II}^{\mathrm{r}-+}$ & $\mathrm{II}^{\mathrm{r}-+}$ & - \\
\hline Origanum vulgare & $\mathrm{I}^{1}$ & $\mathrm{I}^{+}$ & $\mathrm{I}^{1}$ & $\mathrm{I}^{+}$ & $\mathrm{I}^{+-1}$ & - \\
\hline Chamaecytisus ruthenicus & $\mathrm{II}^{+-1}$ & - & $+^{+}$ & $\mathrm{I}^{+}$ & $\mathrm{I}^{+-1}$ & - \\
\hline Poa angustifolia var. setacea & $\mathrm{V}^{+-1}$ & $\mathrm{~V}^{+-2}$ & $\mathrm{~V}^{+-2}$ & $\mathrm{IV}^{\mathrm{r}-+}$ & $\mathrm{V}^{\mathrm{r}-2}$ & $\mathrm{IV}^{1-3}$ \\
\hline Galium verum fo. & $\mathrm{V}^{+-1}$ & $\mathrm{~V}^{+-2}$ & $\mathrm{IV}^{+-2}$ & $\mathrm{I}^{1}$ & $\mathrm{IV}^{+-2}$ & $\mathrm{IV}^{1-2}$ \\
\hline Hypericum perforatum & $\mathrm{III}^{+}$ & $\mathrm{III}^{\mathrm{r}-+}$ & $\mathrm{IIII}^{\mathrm{r}-+}$ & $\mathrm{V}^{+}$ & $\mathrm{IV}^{\mathrm{r}-+}$ & $\mathrm{V}^{+-1}$ \\
\hline Agrimonia procera & $\mathrm{IV}^{\mathrm{r}-+}$ & $\mathrm{IV}^{\mathrm{r}-1}$ & - & $\mathrm{IV}^{+-1}$ & $\mathrm{III}^{\mathrm{r}-1}$ & $\mathrm{~V}^{1-2}$ \\
\hline Galium album fo. & $\mathrm{II}^{\mathrm{r-}-}$ & $\mathrm{II}^{+}$ & $\mathrm{IV}^{+-1}$ & $\mathrm{II}^{+-1}$ & $\mathrm{IIII}^{\mathrm{r}-1}$ & $\mathrm{IV}^{+-2}$ \\
\hline Knautia arvensis fo. & $\mathrm{IV}^{+-1}$ & $\mathrm{IV}^{\mathrm{r}-+}$ & $\mathrm{I}^{+}$ & $\mathrm{I}^{+}$ & $\mathrm{III}^{\mathrm{r}-1}$ & $\mathrm{IV}^{+-1}$ \\
\hline Coronilla varia & $\mathrm{IIII}^{\mathrm{r}-+}$ & $\mathrm{I}^{+}$ & $\mathrm{III}^{\mathrm{r}-1}$ & $\mathrm{II}^{+-1}$ & $\mathrm{III}^{\mathrm{r}-1}$ & $\mathrm{IV}^{+-3}$ \\
\hline Viola hirta & $\mathrm{IV}^{\mathrm{r}-1}$ & $\mathrm{III}^{+-1}$ & $\mathrm{II}^{+-1}$ & $\mathrm{I}^{+}$ & $\mathrm{III}^{\mathrm{r}-1}$ & $\mathrm{II}^{+}$ \\
\hline Clinopodium vulgare & $\mathrm{II}^{+-1}$ & $\mathrm{I}^{1}$ & $\mathrm{III}^{+-2}$ & $\mathrm{III}^{+}$ & $\mathrm{II}^{+-2}$ & $\mathrm{III}^{+-2}$ \\
\hline Senecio jacobaea fo. & $+^{r}$ & $+^{\mathrm{r}}$ & $+^{r}$ & - & $+^{r}$ & $\mathrm{III}^{+-1}$ \\
\hline Fragaria vesca & $\mathrm{I}^{+}$ & - & - & - & $\mathrm{r}^{+}$ & $\mathrm{I}^{+}$ \\
\hline *Campanula persicifolia $(\mathrm{Q}-\mathrm{F})$ & - & - & $+^{+}$ & - & $+^{+}$ & - \\
\hline *Hieracium murorum $(\mathrm{O})$ & - & - & $+^{+}$ & - & $\mathrm{r}^{+}$ & - \\
\hline Vicia sepium & - & - & $+^{r}$ & - & $\mathrm{r}^{\mathrm{r}}$ & - \\
\hline Silene nutans & - & $+^{+}$ & - & - & $\mathrm{r}^{+}$ & - \\
\hline \multicolumn{7}{|l|}{ VII. Ch. Molinio-Arrhenatheretea } \\
\hline Veronica chamaedrys & $\mathrm{II}^{\mathrm{r}-+}$ & $\mathrm{II}^{\mathrm{r}-+}$ & $\mathrm{IV}^{\mathrm{r}-1}$ & $\mathrm{IV}^{\mathrm{r}-+}$ & $\mathrm{III}^{\mathrm{r}-1}$ & $\mathrm{I}^{+}$ \\
\hline Festuca rubra & $\mathrm{I}^{+}$ & $\mathrm{III}^{+-2}$ & $\mathrm{I}^{+}$ & - & $\mathrm{I}^{+-2}$ & - \\
\hline Festuca pratensis & - & $\mathrm{I}^{+-1}$ & $+^{+}$ & - & $+^{+-1}$ & - \\
\hline Centaurea jacea & $\mathrm{I}^{+}$ & - & $+^{+}$ & - & $+^{+}$ & - \\
\hline Vicia cracca & $\mathrm{I}^{+}$ & - & $+^{+}$ & - & $\mathrm{r}^{+}$ & - \\
\hline Climacium dendroides $\mathrm{d}$ & - & $+^{+}$ & $+^{+}$ & - & $\mathrm{r}^{+}$ & - \\
\hline Stachys officinalis & $\mathrm{I}^{2}$ & - & - & - & $\mathrm{I}^{2}$ & - \\
\hline Arrhenatherum elatius & $\mathrm{V}^{+-2}$ & $\mathrm{~V}^{+-2}$ & $\mathrm{~V}^{+-3}$ & $\mathrm{~V}^{+-2}$ & $\mathrm{~V}^{+-3}$ & $\mathrm{~V}^{1-3}$ \\
\hline Dactylis glomerata & $\mathrm{IV}^{+}$ & $\mathrm{III}^{+}$ & $\mathrm{II}^{+}$ & $\mathrm{III}^{+}$ & $\mathrm{III}^{+}$ & $\mathrm{IV}^{+-2}$ \\
\hline Leontodon hispidus & $\mathrm{II}^{\mathrm{r}-+}$ & $\mathrm{I}^{+}$ & $\mathrm{I}^{\mathrm{r}-+}$ & - & $\mathrm{I}^{+}$ & $\mathrm{I}^{+}$ \\
\hline Carex hirta & $\mathrm{II}^{+}$ & $+^{+}$ & $\mathrm{I}^{+}$ & - & $\mathrm{I}^{+}$ & $\mathrm{I}^{1}$ \\
\hline Plantago lanceolata & $\mathrm{III}^{+}$ & $\mathrm{III}^{\mathrm{r}-+}$ & $\mathrm{II}^{\mathrm{r}-+}$ & $\mathrm{I}^{+}$ & $\mathrm{II}^{\mathrm{r}-+}$ & $\mathrm{IV}^{+-1}$ \\
\hline Achillea millefolium & $\mathrm{II}^{\mathrm{r}-+}$ & - & - & - & $+^{r-+}$ & $\mathrm{IV}^{1-2}$ \\
\hline Poa pratensis & - & - & - & - & - & $\mathrm{II}^{1-2}$ \\
\hline Potentilla reptans & - & - & - & - & - & $\mathrm{II}^{1}$ \\
\hline Rumex acetosa & - & - & - & - & - & $\mathrm{II}^{+}$ \\
\hline
\end{tabular}




\begin{tabular}{|c|c|c|c|c|c|c|}
\hline Successive No. & 1 & 2 & 3 & 4 & 5 & 6 \\
\hline Abbreviated syntaxon name & $\mathrm{P}$ & $\mathrm{C}-\mathrm{V}$ & G-A & $\mathrm{L}-\mathrm{V}$ & G coll. & TS \\
\hline Number of relevés & 10 & 12 & 16 & 5 & 43 & 7 \\
\hline Crepis biennis & - & - & - & - & - & $\mathrm{I}^{1}$ \\
\hline Inula salicina & - & - & - & - & - & $\mathrm{I}^{1}$ \\
\hline Briza media & $\mathrm{II}^{1-3}$ & - & - & - & $\mathrm{I}^{1-3}$ & $\mathrm{I}^{1}$ \\
\hline Taraxacum officinale coll. & - & $+^{\mathrm{r}}$ & $\mathrm{I}^{\mathrm{r}}$ & - & $t^{r}$ & $\mathrm{I}^{+}$ \\
\hline Trifolium pratense & - & $\mathrm{I}^{\mathrm{r}-+}$ & $+^{+}$ & - & $++^{\mathrm{r}-+}$ & $\mathrm{I}^{+}$ \\
\hline Lotus corniculatus & $\mathrm{I}^{+}$ & - & - & - & $\mathrm{r}^{+}$ & $\mathrm{I}^{1}$ \\
\hline Prunella vulgaris & - & $\mathrm{I}^{\mathrm{r}-+}$ & - & - & $\mathrm{r}^{\mathrm{r}-\mathrm{+}}$ & - \\
\hline Lathyrus pratensis & - & - & $\mathrm{I}^{+}$ & - & $\mathrm{r}^{+}$ & - \\
\hline Avenula pubescens & - & - & $+^{+}$ & - & $\mathrm{r}^{+}$ & - \\
\hline Carex spicata & - & $+^{+}$ & - & - & $\mathrm{r}^{+}$ & - \\
\hline \multicolumn{7}{|l|}{ VIII. Ch. Koelerio-Corynephoretea } \\
\hline Trifolium campestre & - & - & - & - & - & $\mathrm{IV}^{+-1}$ \\
\hline Cerastium arvense & - & - & - & - & - & $\mathrm{II}^{1}$ \\
\hline Sedum acre & $\mathrm{I}^{+}$ & - & $+^{+}$ & - & $+^{+}$ & $\mathrm{I}^{+}$ \\
\hline Trifolium arvense & - & $+^{\mathrm{r}}$ & - & - & $\mathrm{r}^{\mathrm{r}}$ & $\mathrm{I}^{2}$ \\
\hline Sedum sexangulare & $\mathrm{I}^{+}$ & - & - & - & $\mathrm{r}^{+}$ & - \\
\hline \multicolumn{7}{|l|}{ IX. Ch. Artemisietea vulgaris } \\
\hline Glechoma hederacea & - & $\mathrm{I}^{\mathrm{r}}$ & $\mathrm{I}^{1}$ & $\mathrm{I}^{+}$ & $\mathrm{I}^{\mathrm{r}-1}$ & - \\
\hline Linaria vulgaris & - & $\mathrm{II}^{+}$ & $+^{\mathrm{r}}$ & - & $\mathrm{II}^{\mathrm{r}-+}$ & $\mathrm{II}^{+}$ \\
\hline Elymus repens & $+^{\mathrm{r}}$ & - & - & $\mathrm{II}^{\mathrm{r}-+}$ & $++^{\mathrm{r}-+}$ & $\mathrm{II}^{+-1}$ \\
\hline Convolvulus arvensis & $\mathrm{I}^{\mathrm{r}}$ & $\mathrm{III}^{\mathrm{r}-+}$ & $\mathrm{II}^{+}$ & $\mathrm{II}^{+-1}$ & $\mathrm{II}^{\mathrm{r}-1}$ & $\mathrm{IV}^{+}$ \\
\hline Picris hieracioides & $\mathrm{I}^{+}$ & $\mathrm{II}^{\mathrm{r}-+}$ & - & - & $\mathrm{I}^{\mathrm{r}-+}$ & $\mathrm{III}^{+-1}$ \\
\hline Echium vulgare & - & - & $+^{\mathrm{r}}$ & - & $\mathrm{r}^{\mathrm{r}}$ & $\mathrm{III}^{+-1}$ \\
\hline Carduus acanthoides & - & - & - & - & - & $\mathrm{III}^{+-1}$ \\
\hline Anchusa officinalis & - & - & - & - & - & $\mathrm{I}^{+}$ \\
\hline Cirsium vulgare & - & - & - & - & - & $\mathrm{I}^{+}$ \\
\hline Daucus carota & - & $\mathrm{II}^{+}$ & - & - & $+^{+}$ & - \\
\hline Artemisia vulgaris & - & $\mathrm{I}^{\mathrm{r}-+}$ & - & - & $\mathrm{r}^{\mathrm{r}-\mathrm{+}}$ & - \\
\hline Cichorium intybus & - & $+^{+}$ & $+^{+}$ & - & $\mathrm{r}^{+}$ & - \\
\hline Melandrium album & - & $+^{\mathrm{r}}$ & - & - & $\mathrm{r}^{+}$ & - \\
\hline Pastinaca sativa & - & $+^{+}$ & - & - & $\mathrm{r}^{+}$ & - \\
\hline Euphorbia esula & - & $+^{\mathrm{r}}$ & - & - & $\mathrm{r}^{\mathrm{r}}$ & - \\
\hline \multicolumn{7}{|l|}{ X. Ch. Rhamno-Prunetea } \\
\hline Ligustrum vulgare $\mathrm{b} / \mathrm{c}$ & $\mathrm{IV}^{+-1}$ & $\mathrm{~V}^{+-1}$ & $\mathrm{IV}^{+-2}$ & $\mathrm{II}^{+}$ & $\mathrm{IV}^{+-2}$ & $+^{+}$ \\
\hline Cornus sanguinea b/c & $\mathrm{IV}^{+-2}$ & $\mathrm{IV}^{+-1}$ & $\mathrm{III}^{+-2}$ & $\mathrm{II}^{+-1}$ & $\mathrm{IV}^{+-2}$ & $\mathrm{I}^{1}$ \\
\hline Rosa dumalis b/c & $\mathrm{II}^{\mathrm{r}-+}$ & $\mathrm{III}^{+}$ & $\mathrm{I}^{+}$ & $\mathrm{III}^{+}$ & $\mathrm{II}^{\mathrm{r}-+}$ & - \\
\hline Prunus spinosa b/c & $\mathrm{V}^{+-2}$ & $\mathrm{~V}^{+-3}$ & $\mathrm{~V}^{+-2}$ & $\mathrm{IV}^{+}$ & $\mathrm{V}^{+-3}$ & $\mathrm{III}^{+-2}$ \\
\hline Rhamnus catharticus $\mathrm{b} / \mathrm{c}$ & $\mathrm{III}^{+}$ & $\mathrm{III}^{+-1}$ & $\mathrm{III}^{+-2}$ & $\mathrm{III}^{+}$ & $\mathrm{III}^{+-2}$ & $\mathrm{II}^{+-2}$ \\
\hline Rosa canina $\mathrm{b} / \mathrm{c}$ & $\mathrm{II}^{+-1}$ & $\mathrm{III}^{+}$ & $\mathrm{III}^{+-1}$ & $\mathrm{IV}^{+}$ & $\mathrm{III}^{+-1}$ & $\mathrm{~V}^{+-2}$ \\
\hline Crataegus monogyna b/c & $\mathrm{I}^{+}$ & - & $+^{+}$ & $\mathrm{I}^{+}$ & $+^{+}$ & $\mathrm{II}^{+-1}$ \\
\hline Pyrus pyraster $\mathrm{b} / \mathrm{c}$ & - & $+^{+}$ & $+^{+}$ & $\mathrm{I}^{+}$ & $+^{+}$ & $\mathrm{I}^{+}$ \\
\hline Rosa rubiginosa b/c & - & - & $+^{+}$ & - & $\mathrm{r}^{+}$ & $\mathrm{I}^{+}$ \\
\hline Rosa inodora b/c & - & - & - & - & - & $\mathrm{I}^{1-2}$ \\
\hline Rosa tomentosa $\mathrm{b} / \mathrm{c}$ & - & - & - & - & - & $\mathrm{I}^{+}$ \\
\hline Crataegus rhipidophylla b/c & - & $\mathrm{I}^{\mathrm{r}-+}$ & - & - & $\mathrm{r}^{\mathrm{r}-+}$ & - \\
\hline Viburnum opulus $\mathrm{b} / \mathrm{c}$ & - & $+^{+}$ & - & - & $\mathrm{r}^{+}$ & - \\
\hline Populus tremula $\mathrm{b} / \mathrm{c}$ & $\mathrm{I}^{+}$ & - & - & - & $\mathrm{r}^{+}$ & - \\
\hline Frangula alnus b/c & $+^{\mathrm{r}}$ & - & - & - & $\mathrm{r}^{\mathrm{r}}$ & - \\
\hline \multicolumn{7}{|l|}{ XI. Ch. Querco-Fagetea } \\
\hline Carex montana & $I^{1-2}$ & + & $\mathrm{I}^{+-1}$ & - & $\mathrm{I}^{+-2}$ & - \\
\hline Euonymus verrucosus b/c & $\mathrm{I}^{+}$ & - & $\mathrm{I}^{+-1}$ & - & $\mathrm{I}^{+-1}$ & $\mathrm{I}^{+-1}$ \\
\hline Acer pseudoplatanus b/c & - & $\mathrm{I}^{\mathrm{r}-+}$ & - & - & $\mathrm{r}^{\mathrm{r}-\mathrm{+}}$ & - \\
\hline Carex digitata & - & $+^{+}$ & $+^{+}$ & - & $\mathrm{r}^{+}$ & - \\
\hline Melica nutans & $\mathrm{I}^{+}$ & - & - & - & $\mathrm{r}^{+}$ & - \\
\hline Malus sylvestris $\mathrm{b} / \mathrm{c}$ & - & - & $+^{+}$ & - & $\mathrm{r}^{+}$ & - \\
\hline Ajuga reptans & - & - & $+^{r}$ & - & $\mathrm{r}^{\mathrm{r}}$ & - \\
\hline
\end{tabular}




\begin{tabular}{|c|c|c|c|c|c|c|}
\hline Successive No. & 1 & 2 & 3 & 4 & 5 & 6 \\
\hline Abbreviated syntaxon name & $\mathrm{P}$ & $\mathrm{C}-\mathrm{V}$ & G-A & $\mathrm{L}-\mathrm{V}$ & G coll. & TS \\
\hline Number of relevés & 10 & 12 & 16 & 5 & 43 & 7 \\
\hline Fagus sylvatica b/c & $+^{\mathrm{r}}$ & - & - & - & $\mathrm{r}^{\mathrm{r}}$ & - \\
\hline Digitalis grandiflora & - & - & - & - & - & $\mathrm{I}^{+}$ \\
\hline \multicolumn{7}{|l|}{ XII. Ch. Vaccinio-Piceetea } \\
\hline Juniperus communis b/c & $\mathrm{V}^{+-1}$ & $\mathrm{III}^{+-2}$ & $\mathrm{III}^{+-2}$ & $\mathrm{I}^{+}$ & $\mathrm{III}^{+-2}$ & $\mathrm{IV}^{+-2}$ \\
\hline Pinus sylvestris $\mathrm{b} / \mathrm{c}$ & $\mathrm{II}^{\mathrm{r}-+}$ & $+^{+}$ & $\mathrm{I}^{+}$ & - & $\mathrm{I}^{+}$ & $\mathrm{II}^{+-2}$ \\
\hline Pleurozium schreberi $\mathrm{d}$ & $\mathrm{I}^{+}$ & - & $\mathrm{I}^{+-1}$ & - & $+^{+-1}$ & - \\
\hline Pseudoscleropodium purum $\mathrm{d}$ & $\mathrm{I}^{+}$ & - & $\mathrm{I}^{+-1}$ & - & $+^{+-1}$ & - \\
\hline Dicranum polysetum $\mathrm{d}$ & - & - & $I^{1-2}$ & - & $I^{1-2}$ & - \\
\hline Rubus saxatilis & $\mathrm{I}^{1}$ & - & - & - & $\mathrm{I}^{1}$ & - \\
\hline Melampyrum pratense & - & - & - & - & - & $\mathrm{I}^{2}$ \\
\hline \multicolumn{7}{|l|}{ XIII. Others } \\
\hline Cruciata glabra & $\mathrm{II}^{\mathrm{r}-+}$ & $\mathrm{I}^{1}$ & $\mathrm{III}^{\mathrm{r}-+}$ & - & $\mathrm{II}^{\mathrm{r}-1}$ & - \\
\hline Plagiomnium affine $\mathrm{d}$ & $\mathrm{I}^{+}$ & $+^{+}$ & $\mathrm{II}^{+-3}$ & $\mathrm{I}^{+}$ & $\mathrm{I}^{+-3}$ & - \\
\hline Thuidium philibertii $\mathrm{d}$ & $\mathrm{I}^{+}$ & $\mathrm{I}^{2}$ & $\mathrm{II}^{+-1}$ & - & $\mathrm{I}^{+-2}$ & - \\
\hline Quercus robur b/c & $\mathrm{I}^{+-1}$ & $\mathrm{I}^{+}$ & $\mathrm{I}^{\mathrm{r}-+}$ & $+^{r}$ & $\mathrm{I}^{\mathrm{r}-1}$ & - \\
\hline Hieracium pilosella & $\mathrm{III}^{+}$ & $\mathrm{II}^{\mathrm{r}-1}$ & $+^{+}$ & $\mathrm{I}^{+}$ & $\mathrm{II}^{\mathrm{r}-1}$ & $\mathrm{II}^{+-1}$ \\
\hline Calamagrostis epigejos & - & $+^{+}$ & $\mathrm{I}^{2}$ & $\mathrm{I}^{+}$ & $+^{+-2}$ & $\mathrm{II}^{1}$ \\
\hline Silene vulgaris & - & $+^{\mathrm{r}}$ & $\mathrm{II}^{\mathrm{r}-+}$ & - & $\mathrm{I}^{\mathrm{r}-+}$ & $\mathrm{V}^{1-2}$ \\
\hline Pteridium aquilinum & $\mathrm{I}^{+-2}$ & $+^{+}$ & - & - & $t^{+-2}$ & $\mathrm{III}^{+-2}$ \\
\hline Cerinthe minor & - & - & - & - & - & $\mathrm{II}^{1}$ \\
\hline Geranium columbinum & - & - & - & - & - & $\mathrm{II}^{+-1}$ \\
\hline Malus domestica b/c & - & $\mathrm{I}^{+}$ & - & - & $\mathrm{r}^{+}$ & $\mathrm{I}^{+}$ \\
\hline Oxyrrhynchium hians d & - & $\mathrm{II}^{+-1}$ & $+^{+}$ & - & $+^{+-1}$ & - \\
\hline Geranium pusillum & - & - & $\mathrm{II}^{\mathrm{r}-+}$ & - & $+^{r-+}$ & - \\
\hline Vicia hirsuta & - & - & $\mathrm{II}^{+}$ & - & $+^{+}$ & - \\
\hline Brachythecium rutabulum $\mathrm{d}$ & $\mathrm{I}^{+}$ & $I^{1-2}$ & - & - & $t^{+-2}$ & - \\
\hline Prunus domestica b/c & - & $\mathrm{I}^{+}$ & - & - & $\mathrm{r}^{+}$ & - \\
\hline Stachys annua & - & $+^{\mathrm{r}}$ & - & - & $\mathrm{r}^{\mathrm{r}}$ & - \\
\hline Calliergonella cuspidata $\mathrm{d}$ & - & $\mathrm{I}^{3}$ & - & - & $\mathrm{I}^{3}$ & - \\
\hline Anthoxanthum odoratum & - & $+^{+}$ & - & - & $\mathrm{r}^{+}$ & - \\
\hline Cimicifuga europaea & $\mathrm{I}^{+}$ & - & - & - & $\mathrm{r}^{+}$ & - \\
\hline Fissidens cristatus $\mathrm{d}$ & $\mathrm{I}^{+}$ & - & - & - & $\mathrm{r}^{+}$ & - \\
\hline Fissidens sp. d & - & - & $+^{+}$ & - & $\mathrm{r}^{+}$ & - \\
\hline Tragopogon sp. & $\mathrm{I}^{+}$ & - & - & - & $\mathrm{r}^{+}$ & - \\
\hline Agrostis capillaris & $+^{+}$ & - & - & - & $\mathrm{r}^{+}$ & $\mathrm{I}^{1}$ \\
\hline Quercus petraea b/c & - & - & $+^{r}$ & - & $\mathrm{r}^{\mathrm{r}}$ & $\mathrm{I}^{+-1}$ \\
\hline Polygala vulgaris & - & - & - & - & - & $\mathrm{I}^{+}$ \\
\hline Lychnis viscaria & - & - & - & - & - & $\mathrm{I}^{+}$ \\
\hline Rhinanthus angustifolius & - & - & - & - & - & $\mathrm{I}^{+}$ \\
\hline Sedum maximum & - & - & - & - & - & $\mathrm{I}^{+}$ \\
\hline Myosotis arvensis & - & - & - & - & - & $\mathrm{I}^{+}$ \\
\hline Convallaria majalis & - & - & - & - & - & $\mathrm{I}^{+}$ \\
\hline
\end{tabular}

Explanations: P - Peucedanetum cervariae, C-V - Campanulo bononiensis-Vicietum tenuifoliae, G-A - Geranio-Anemonetum sylvestris, L-V - Lathyro sylvestris-Vincetoxicetum hirundinariae, G coll. - all four associations of the alliance Geranion sanguinei, TS - Thalictro-Salvietum pratensis 
Table 7. Chosen parameters characterising the state of non-forest xerothermic vegetation in the "Murawy Dobromierskie" reseve in the period 1993-2012

\begin{tabular}{lrrrrrc}
\hline Year of study & \multicolumn{3}{c}{1993} & \multicolumn{3}{c}{2012} \\
\hline Parameters & \multicolumn{1}{c}{$\mathrm{C}$} & \multicolumn{1}{c}{$\mathrm{G}$} & \multicolumn{1}{c}{$\mathrm{D}$} & $\mathrm{C}$ & $\mathrm{G}$ & $\mathrm{D}$ \\
\hline Ch. of the examined Geranion sanguinei associations & 2972 & 4,611 & 2,635 & 3089 & 4,886 & 2,219 \\
Ch. Geranion sanguinei & 4186 & $\mathbf{6 , 9 1 6}$ & $\mathbf{4 , 7 4 3}$ & 3458 & 3,562 & 1,259 \\
Ch. Origanetalia, Trifolio-Geranietea sanguinei & $\mathbf{5 8 8 6}$ & 6,34 & 3,321 & 1238 & 5,84 & 2,424 \\
Ch. Cirsio-Brachypodion pinnati & $\mathbf{4 1 7 8}$ & 3,746 & 1,16 & 98 & 4,18 & 1,019 \\
Ch. Brometalia erecti & $\mathbf{2 4 2 2}$ & $\mathbf{2 7 , 0 9}$ & $\mathbf{1 3 , 9 9}$ & 597 & 19,06 & 8,016 \\
Ch. Festuco-Brometea & $\mathbf{1 4 1 7 0}$ & 14,41 & 9,357 & 1415 & 19,07 & 9,101 \\
Ch. Molinio-Arrhenatheretea & $\mathbf{5 6 1 2}$ & 10,66 & 3,523 & 1042 & 12,04 & $\mathbf{6 , 2 9 5}$ \\
Ch. Artemisietea vulgaris & 508 & 2,017 & 0,504 & 442 & 0,286 & 0,035 \\
Ch. Koelerio-Corynephoretea & $\mathbf{4 2 1}$ & $\mathbf{6 , 6 2 8}$ & $\mathbf{2 , 4 2}$ & 5 & 4,666 & 1,038 \\
Ch. Rhamno-Prunetea & $\mathbf{3 1 4 1}$ & 6,628 & 2,178 & 1472 & $\mathbf{1 1 , 6 5}$ & $\mathbf{5 , 3 4 9}$ \\
Others & $\mathbf{2 3 3 5}$ & $\mathbf{8 , 0 6 9}$ & $\mathbf{2 , 1 5 2}$ & 465 & 5,468 & 0,955 \\
\hline
\end{tabular}

Explanations: $\mathrm{C}$ - cover coefficient, $\mathrm{G}$ - collective group share index, D - systematic group value (the values of a greater importance for the studied problem are typed in bold) 